\title{
Bistatic Synthetic Aperture Radar Imaging Using UltraNarrowband Continuous Waveforms
}

\author{
Ling Wang, Member, IEEE, and Birsen Yazici, Senior Member, IEEE
}

\begin{abstract}
We consider synthetic aperture radar (SAR) imaging using ultranarrowband continuous waveforms (CWs). Because of the high Doppler resolution of $\mathrm{CW}$ signals, we refer to this imaging modality as Doppler synthetic aperture radar (DSAR). We present a novel model and an image formation method for the bistatic DSAR for arbitrary imaging geometries. Our bistatic DSAR model is formed by correlating the translated version of the received signal with a scaled or frequency-shifted version of the transmitted $\mathrm{CW}$ signal over a finite time window. High-frequency analysis of the resulting model shows that the correlated signal is the projections of the scene reflectivity onto the bistatic iso-Doppler curves. We next use microlocal techniques to develop a filtered-backprojection (FBP) type image reconstruction method. The FBP inversion results in the backprojection of the correlated signal onto the bistatic iso-Doppler curves as opposed to the bistatic iso-range curves used in the traditional wideband SAR imaging. We show that our method takes advantage of the velocity, as well as the acceleration of the antennas in certain directions, to form a high-resolution SAR image. Our bistatic DSAR imaging method is applicable for arbitrary flight trajectories and nonflat topography, and can accommodate system-related parameters. We present resolution analysis and extensive numerical experiments to demonstrate the performance of our imaging method.
\end{abstract}

Index Terms-Continuous wave (CW), Doppler, filteredbackprojection, imaging, synthetic aperture.

\section{INTRODUCTION}

$\mathbf{T}$ RADITIONAL synthetic aperture radar (SAR) uses wideband waveforms to provide high range resolution. We consider an SAR imaging system that uses ultranarrowband or single-frequency transmitted continuous waveforms (CWs). Because of the high Doppler resolution nature of the transmitted waveforms, we refer to the system under consideration as Doppler synthetic aperture radar (DSAR) or "Doppler SAR" for short.

As compared to the traditional SAR system, Doppler SAR requires a relatively simple and low-cost transmitter, and in

Manuscript received November 27, 2010; revised January 25, 2012; accepted March 15, 2012. Date of publication April 3, 2012; date of current version July 18, 2012. This work was supported in part by the Air Force Office of Scientific Research under Agreement FA9550-07-1-0363 and Agreement FA9550-09-1-0013 and in part by the National Science Foundation under Grant CCF-08030672. The associate editor coordinating the review of this manuscript and approving it for publication was Prof. Brian D. Rigling.

L. Wang is with the Department of Information and Communication Engineering, Nanjing University of Aeronautics and Astronautics, Nanjing 210016, China (e-mail: wanglrpi@gmail.com).

B. Yazici is with the Department of Electrical, Computer and Systems Engineering, Rensselaer Polytechnic Institute, Troy, NY 12180 USA (e-mail: yazici@ecse.rpi.edu).

Color versions of one or more of the figures in this paper are available online at http://ieeexplore.ieee.org.

Digital Object Identifier 10.1109/TIP.2012.2193134 some cases it does not even need a dedicated transmitter. Existing radio frequency signals, such as radio and television signals and cellphone signals, can be used as the illumination sources for Doppler SAR. These waveforms are continuous, ultranarrowband, and have high Doppler resolution. In this paper, we present a novel filtered-backprojection (FBP) method for bistatic Doppler SAR with arbitrary imaging geometries.

Radar imaging with ultranarrowband waveforms has been presented in the literature earlier [1]-[9]. There are mainly two approaches to radar imaging using ultranarrowband waveforms. The first approach is based on trading the resolution provided by high bandwidth with the resolution provided by the spatial diversity [1]-[5]. In [1] and [2], the imaging of rotating objects using a single-frequency continuous wave illumination was investigated theoretically, and in [3] studied experimentally. In [4] and [5], the imaging of stationary or slow-moving objects using ultranarrowband or narrowband CW signals with spatially distributed radars was studied. In [4], a practical tomographic approach for target detection using ultranarrowband transmissions from a multistatic radar was presented. It was shown that the method can provide high-resolution surveillance of a region of interest. In [5], a multistatic narrowband $\mathrm{CW}$ radar system illuminating a target over 360 degrees of aspect angles was presented. It was shown that such a system can achieve spatial resolution superior to those of SAR or inverse synthetic aperture radar systems using wideband waveforms. In all these studies, the imaging method images scatterers that lie on iso-range curves.

The second approach to ultranarrowband radar imaging relies on the idea of imaging scatterers that lie on iso-Doppler curves [6]-[9]. The work presented in this paper falls into this category. This idea was first introduced in [6]. In [7], the idea of imaging scatterers lying on iso-Doppler contours was introduced to map polar regions of terrestrial planets using the Doppler shifts of the received signal. The data was collected for several repeated passes over the polar region. During each pass, the antenna was assumed to be traversing a straight flight trajectory at a constant height, with a constant velocity over a flat topography. Consequently, the iso-Doppler contours considered were weighted hyperbolas. An FBP-type inversion formula was presented to recover the surface reflectivity from its projections onto weighted hyperbolas. The resolution of the imaging method was presented, along with its discrete implementation. In [8], the same idea introduced in [6] and [7] was explored for the monostatic SAR. An FBP method to recover the ground reflectivity from its projections onto hyperbolas for the straight flight trajectory and flat 
topography was presented. An expedient and approximate implementation of the method based on detecting curves associated with scatterers using the Radon-Hough transform was presented. In [9], we explored the idea of imaging scatterers on iso-Doppler curves for passive synthetic aperture imaging using ultranarrowband waveforms and introduced a new SAR modality. Our work introduced the concept of passive Doppler-scale factor and corresponding passive iso-Doppler curves. The image reconstruction was performed via an FBP-type inversion. This paper is particularly suitable for non-cooperative transmitters of opportunity where the locations of the transmitting antennas are unknown.

In this paper, we present a novel imaging method for bistatic DSAR, where the receiving and transmitting antennas are sufficiently far apart. Our method is not restricted to a particular geometry and includes arbitrary, but known, flight trajectories and nonflat topography. The imaging method consists of a novel forward model for bistatic DSAR and a corresponding novel image formation method. We first correlate the translated version of the received signal with a scaled or frequencyshifted version of the transmitted signal over a finite time window. The high-frequency analysis of the resulting model shows that the correlated received signal is the projections of the scene reflectivity onto the bistatic iso-Doppler curves. Our analysis shows that the spread of the iso-Doppler contours on the ground is directly related to the Doppler ambiguity of the transmitted waveforms. We next use microlocal techniques to develop an FBP-type inversion method to reconstruct the scene reflectivity. The analysis of the point spread function (PSF) of the imaging operator shows that the reconstructed images preserve the location and orientation of the singularities at the intersection of the bistatic iso-Doppler and iso-Doppler rate curves introduced in this paper. This means that the our imaging scheme takes advantage of the velocity as well as the acceleration of the antennas in certain directions to form a high-resolution SAR image using ultranarrowband waveforms. The final image is formed by the superposition of the images obtained over a range of translated time windows.

We show that the resolution of the image is directly related to the length of the support of the windowing function, the carrier-frequency of the transmitted waveform, the sampling rate of the aperture, the number of the time windows used for imaging, the velocities of the transmitter and receiver, and the range of the antennas to the scatterers. We describe algorithmic implementation and computational complexity of our reconstruction method and present extensive numerical experiments to validate the theoretical analysis and to demonstrate the performance of our imaging method.

Our imaging method has the following advantages.

1) It can be used for arbitrary imaging geometries including bistatic geometry, arbitrary flight trajectories and nonflat topography.

2) It can be used for passive synthetic aperture imaging for cooperative sources of opportunity where the locations of the transmitting antennas are known. However, unlike some of the existing cooperative passive imaging techniques [10]-[17], it does not require antennas with high directivity.
3) It can be used with both stationary and/or mobile transmitters.

4) It has the desirable property of preserving the visible edges of the scene in the reconstructed image.

5) It is an analytic reconstruction technique which can be made computationally efficient [18].

The organization of this paper is as follows. In Section II-A, we present the model for the received signal. In Section II-B, we develop the forward model for bistatic DSAR. In Section II-C, we analyze the critical points of our forward model. In Section III, we develop an FBP-type image formation method for the bistatic DSAR, analyze the resolution of DSAR imaging, and present the algorithmic implementation and computational complexity of our image reconstruction method. In Section IV, we present numerical simulations. Section V concludes this paper.

\section{Bistatic DSAR MEAsurement Model}

In this section, we first describe the received signal model for a moving transmitter using a $\mathrm{CW}$ and then derive a forward model by correlating the windowed and translated received signal with the windowed scaled or frequency-shifted transmitted waveform. The resulting processed signal is then used to form an image of the scene by an FBP method.

\section{A. Model for the Received Signal}

We use the following notational conventions throughout this paper. Bold Roman, bold Italic, and Roman lower-case letters are used to denote variables in $\mathbb{R}^{3}, \mathbb{R}^{2}$, and $\mathbb{R}$, respectively, i.e., $\mathbf{z}=\left(z, z_{3}\right) \in \mathbb{R}^{3}$, with $z=\left(z_{1}, z_{2}\right) \in \mathbb{R}^{2}$ and $z_{i}, i=$ $1,2,3 \in \mathbb{R}$. Calligraphic letters $(\mathcal{F}, \mathcal{K}$, etc., $)$ are used to denote operators.

For a pair of transmitter and receiver antennas located at $\mathbf{T}$ and $\mathbf{R}$, respectively, the received signal is modeled by [19]

$$
\begin{aligned}
f(t, \mathbf{R}, \mathbf{T})= & \int \frac{\mathrm{e}^{\mathrm{i} \omega\left(\mathrm{t}-(|\mathbf{R}-\mathbf{z}|+|\mathbf{z}-\mathbf{T}|) / \mathrm{c}_{0}\right)}}{(4 \pi)^{2}|\mathbf{R}-\mathbf{z}||\mathbf{z}-\mathbf{T}|} \omega^{2} \hat{p}(\omega) \\
& \times J_{\operatorname{tr}}(\omega, \mathbf{z}, \mathbf{T}) J_{\mathrm{rc}}(\omega, \mathbf{z}, \mathbf{R}) V(\mathbf{z}) d \omega d \mathbf{z}
\end{aligned}
$$

where $t$ denotes time, $\hat{p}$ denotes the Fourier transform of the transmitted waveform $p(t), c_{0}$ denotes the speed of light in free space, $V(\mathbf{z})$ is the reflectivity function, and $J_{\text {tr }}$ and $J_{\text {rc }}$ are the transmitter and receiver antenna beampattern related terms, respectively.

Let $\mathbf{z}=(\boldsymbol{z}, \boldsymbol{\psi}(\boldsymbol{z})) \in \mathbb{R}^{3}$ denote the Earth's surface, where $z \in \mathbb{R}^{2}$ and $\boldsymbol{\psi}: \mathbb{R}^{2} \rightarrow \mathbb{R}$ are known functions of the ground topography. Furthermore, we assume that the scattering takes place in a thin region near the surface. Thus, the reflectivity function has the following form:

$$
V(\mathbf{z})=\rho(z) \delta\left(z_{3}-\boldsymbol{\psi}(\boldsymbol{z})\right) .
$$

For a narrowband waveform, we have

$$
p(t)=\mathrm{e}^{\mathrm{i} \omega_{0} \mathrm{t}} \widetilde{p}(\mathrm{t})
$$

where $\omega_{0}$ denotes the angular carrier frequency and $\tilde{p}(t)$ is the complex envelope of $p$, which is slow varying as a function of $t$ as compared to $\mathrm{e}^{\mathrm{i} \omega_{0} \mathrm{t}}$. 
Using (2) and the Fourier transform of (3), and under the assumption of broadband antenna, after rearranging the terms, (1) becomes

$$
\begin{aligned}
f(t, \mathbf{R}, \mathbf{T}) \approx & \int \mathrm{e}^{\mathrm{i} \omega_{0}\left(\mathrm{t}-(|\mathbf{R}-\mathbf{z}|+|\mathbf{z}-\mathbf{T}|) / \mathrm{c}_{0}\right)} \\
& \times \tilde{p}\left(t-(|\mathbf{R}-\mathbf{z}|+|\mathbf{z}-\mathbf{T}|) / c_{0}\right) \\
& \times A_{\mathrm{TR}}\left(\omega_{0}, \mathbf{z}, \mathbf{R}, \mathbf{T}\right) \rho(\boldsymbol{z}) d \boldsymbol{z}
\end{aligned}
$$

where $A_{\mathrm{TR}}$ is given by

$$
\begin{aligned}
A_{\mathrm{TR}}\left(\omega_{0}, \mathbf{z}, \mathbf{R}, \mathbf{T}\right)= & \frac{\omega_{0}^{2}}{(4 \pi)^{2}|\mathbf{R}-\mathbf{z}||\mathbf{z}-\mathbf{T}|} \\
& \times J_{\mathrm{tr}}\left(\omega_{0}, \mathbf{z}, \mathbf{T}\right) J_{\mathrm{rc}}\left(\omega_{0}, \mathbf{z}, \mathbf{R}\right) .
\end{aligned}
$$

Note that for the rest of this paper, unless otherwise stated, we use $\mathbf{z}=(z, \psi(z))$.

For the rest of our discussion, we consider bistatic synthetic aperture radar (BISAR) imaging. Let $\boldsymbol{\gamma}_{T}(t)$ and $\boldsymbol{\gamma}_{R}(t)$ be the transmitter and receiver trajectories, respectively, and $s(t)$ denote the received signal. We then have

$$
s(t)=f\left(t, \boldsymbol{\gamma}_{R}(t), \boldsymbol{\gamma}_{T}(t)\right) .
$$

Using (4), the received signal for BISAR transmitting an ultranarrowband $\mathrm{CW}$ becomes

$$
\begin{aligned}
s(t)= & \int \mathrm{e}^{\mathrm{i} \omega_{0}\left(t-R_{\mathrm{TR}}(t, \boldsymbol{z}) / c_{0}\right)} \tilde{p}\left(t-R_{\mathrm{TR}}(t, \boldsymbol{z}) / c_{0}\right) \\
& \times A_{\mathrm{TR}}\left(\omega_{0}, \mathbf{z}, \boldsymbol{\gamma}_{R}(t), \boldsymbol{\gamma}_{T}(t)\right) \rho(\boldsymbol{z}) d \boldsymbol{z}
\end{aligned}
$$

where

$$
R_{\mathrm{TR}}(t, \boldsymbol{z})=\left|\boldsymbol{\gamma}_{T}(t)-\mathbf{z}\right|+\left|\mathbf{z}-\boldsymbol{\gamma}_{R}(t)\right|
$$

is the bistatic range and $A_{\mathrm{TR}}\left(\omega_{0}, \mathbf{z}, \boldsymbol{\gamma}_{R}(t), \boldsymbol{\gamma}_{T}(t)\right)$ is given by (5).

\section{B. Forward Model for Bistatic DSAR}

We now use the received signal model presented in the previous subsection to develop a forward model that facilitates imaging of scatterers for which the moving receivers observe a constant Doppler-scaling factor. To form the forward model, we first divide the received signal into different windows. We then translate and correlate the windowed received signal with a scaled and shifted version of the transmitted signal. The scaling allows us to determine the location of the scatters on the stationary ground for which the moving receivers observe a constant Doppler-scaling factor in certain directions. This processing of the received signal and the resulting forward model are described in detail below.

We define the correlation of the received signal given by (7) with a scaled or frequency-shifted version ${ }^{1}$ of the transmitted signal over a finite time window by

$$
d\left(\tau^{\prime}, \tau, \mu\right)=\int s(t+\tau) p^{*}(\mu t) \phi\left(t-\tau^{\prime}\right) d t
$$

for some $\tau, \tau^{\prime} \in \mathbb{R}$, and $\mu \in \mathbb{R}^{+}$, where $\phi\left(t-\tau^{\prime}\right)$ is a smooth windowing function centered at $t=\tau^{\prime}$ with finite support.

\footnotetext{
${ }^{1}$ Note that, since the bandwidth of a narrowband waveform is much smaller than its carrier frequency, the scaled version of the waveform is approximately equal to its frequency-shifted version.
}

Note that (9) can be viewed as "Doppler-based matched filtering." The parameter $\tau$ is used to divide the received signal into windows, and the parameter $\mu$ is used to account for the Doppler frequency within each processed window. The parameter $\tau^{\prime}$ is introduced to shift the scaled transmitted signal prior to correlation. In the following discussion, we develop a mapping that relates the data $d\left(\tau^{\prime}, \tau, \mu\right)$ to the scene to be imaged.

Substituting (7) into (9), we have

$$
\begin{aligned}
d\left(\tau^{\prime}, \tau, \mu\right)= & \int \mathrm{e}^{\mathrm{i} \omega_{0}\left(t+\tau-R_{\mathrm{TR}}(t+\tau, \boldsymbol{z}) / c_{0}\right)} \mathrm{e}^{-\mathrm{i} \omega_{0} \mu \mathrm{t}} \\
& \times \tilde{p}\left(t+\tau-R_{\mathrm{TR}}(t+\tau, \boldsymbol{z}) / c_{0}\right) \tilde{p}^{*}(\mu t) \\
& \times A_{\mathrm{TR}}\left(\omega_{0}, \mathbf{z}, \boldsymbol{\gamma}_{R}(t+\tau), \boldsymbol{\gamma}_{T}(t+\tau)\right) \\
& \times \rho(\boldsymbol{z}) d \boldsymbol{z} \phi\left(t-\tau^{\prime}\right) d t
\end{aligned}
$$

where $R_{\mathrm{TR}}(t+\tau, \boldsymbol{z})$ is defined by (8) with the time-variable $t$ replaced with $t+\tau$.

We make the change of variables $t^{\prime}=t-\tau^{\prime}$ in (10) and obtain

$$
\begin{aligned}
d\left(\tau^{\prime}, \tau, \mu\right)= & \int \mathrm{e}^{\mathrm{i} \omega_{0}\left(t^{\prime}+\tau^{\prime}+\tau-R_{\mathrm{TR}}\left(t^{\prime}+\tau^{\prime}+\tau, \boldsymbol{z}\right) / c_{0}\right)} \mathrm{e}^{-\mathrm{i} \omega_{0} \mu\left(\mathrm{t}^{\prime}+\tau^{\prime}\right)} \\
& \times \tilde{p}\left(t^{\prime}+\tau^{\prime}+\tau-R_{\mathrm{TR}}\left(t^{\prime}+\tau^{\prime}+\tau, \boldsymbol{z}\right) / c_{0}\right) \\
& \times \tilde{p}^{*}\left(\mu\left(t^{\prime}+\tau^{\prime}\right)\right) \\
& \times A_{\mathrm{TR}}\left(\omega_{0}, \mathbf{z}, \boldsymbol{\gamma}_{R}\left(t^{\prime}+\tau^{\prime}+\tau\right), \boldsymbol{\gamma}_{T}\left(t^{\prime}+\tau^{\prime}+\tau\right)\right) \\
& \times \rho(\boldsymbol{z}) d \boldsymbol{z} \phi\left(t^{\prime}\right) d t^{\prime}
\end{aligned}
$$

For notational simplicity, we replace $t^{\prime}$ with $t$ and write

$$
\begin{aligned}
A_{\tilde{p}}\left(\mathbf{z}, t, \tau^{\prime}, \tau, \mu\right)=\tilde{p}(t & \left.+\tau^{\prime}+\tau-R_{\mathrm{TR}}\left(t+\tau^{\prime}+\tau, \boldsymbol{z}\right) / c_{0}\right) \\
& \times \tilde{p}^{*}\left(\mu\left(t+\tau^{\prime}\right)\right) \\
A_{\mathrm{TR}}\left(\omega_{0}, \mathbf{z}, t, \tau^{\prime}, \tau\right)= & A_{\mathrm{TR}}\left(\omega_{0}, \mathbf{z}, \boldsymbol{\gamma}_{R}\left(t+\tau^{\prime}+\tau\right),\right. \\
& \left.\times \boldsymbol{\gamma}_{T}\left(t+\tau^{\prime}+\tau\right)\right) .
\end{aligned}
$$

Thus, (11) becomes

$$
\begin{aligned}
d\left(\tau^{\prime}, \tau, \mu\right)= & \int \mathrm{e}^{\mathrm{i} \omega_{0}\left(t+\tau^{\prime}+\tau-R_{\mathrm{TR}}\left(t+\tau^{\prime}+\tau, \boldsymbol{z}\right) / c_{0}\right)} \mathrm{e}^{-\mathrm{i} \omega_{0} \mu\left(\mathrm{t}+\tau^{\prime}\right)} \\
& \times A_{\tilde{p}}\left(\mathbf{z}, t, \tau^{\prime}, \tau, \mu\right) A_{\mathrm{TR}}\left(\omega_{0}, \mathbf{z}, t, \tau^{\prime}, \tau\right) \\
& \times \rho(\boldsymbol{z}) d \boldsymbol{z} \phi(t) d t
\end{aligned}
$$

Now using the Taylor expansion of $\boldsymbol{\gamma}_{T}\left(t+\tau^{\prime}+\tau\right)$ and $\gamma_{R}\left(t+\tau^{\prime}+\tau\right)$ at $t=0$, for a relatively short time window, and under the assumptions that $\dot{\boldsymbol{\gamma}}_{T}\left(\tau^{\prime}+\tau\right) t \ll\left|\boldsymbol{\gamma}_{T}\left(\tau^{\prime}+\tau\right)-\mathbf{z}\right|$ and $\dot{\gamma}_{R}\left(\tau^{\prime}+\tau\right) t \ll\left|\boldsymbol{\gamma}_{R}\left(\tau^{\prime}+\tau\right)-\mathbf{z}\right|$, we approximate

$$
\begin{aligned}
\left|\boldsymbol{\gamma}_{T}\left(t+\tau^{\prime}+\tau\right)-\mathbf{z}\right| \approx & \left|\boldsymbol{\gamma}_{T}\left(\tau^{\prime}+\tau\right)-\mathbf{z}\right|+\left(\boldsymbol{\gamma}_{T}\left(\widehat{\tau^{\prime}+\tau}\right)-\mathbf{z}\right) \\
& \dot{\boldsymbol{\gamma}}_{T}\left(\tau^{\prime}+\tau\right) t
\end{aligned}
$$

and

$$
\begin{aligned}
\left|\mathbf{z}-\boldsymbol{\gamma}_{R}\left(t+\tau^{\prime}+\tau\right)\right| \approx & \left.\left|\mathbf{z}-\boldsymbol{\gamma}_{R}\left(\tau^{\prime}+\tau\right)\right|-\left(\mathbf{z}-\widehat{\boldsymbol{\gamma}_{R}\left(\tau^{\prime}\right.}+\tau\right)\right) \\
& \dot{\boldsymbol{\gamma}}_{R}\left(\tau^{\prime}+\tau\right) t
\end{aligned}
$$

where $\hat{\mathbf{z}}$ denotes the unit vector in the direction of $\mathbf{z}$. Note that in practical scenarios, (15) and (16) are always satisfied for antennas sufficiently away from the illuminated scene. 
Substituting (15) and (16) into (14), and rearranging the terms, we obtain

$$
\begin{aligned}
d\left(\tau^{\prime}, \tau, \mu\right) \approx & \int \mathrm{e}^{\mathrm{i} 2 \pi t\left[f_{0}(1-\mu)-f_{d}\left(\tau^{\prime}, \tau, \mathbf{z}\right)\right]} \\
& \times \mathrm{e}^{\mathrm{i} \omega_{0}\left[(1-\mu) \tau^{\prime}+\tau-R_{\mathrm{TR}}\left(\tau^{\prime}+\tau, \boldsymbol{z}\right) / c_{0}\right]} \\
& \times A_{\tilde{p}}\left(\mathbf{z}, t, \tau^{\prime}, \tau, \mu\right) A_{\mathrm{TR}}\left(\omega_{0}, \mathbf{z}, t, \tau^{\prime}, \tau\right) \\
& \times \rho(\boldsymbol{z}) d \boldsymbol{z} \phi(t) d t
\end{aligned}
$$

where $f_{0}=\omega_{0} /(2 \pi)$ and

$$
\begin{aligned}
f_{d}\left(\tau^{\prime}, \tau, \mathbf{z}\right)=\frac{f_{0}}{c_{0}}[ & \left(\boldsymbol{\gamma}_{T}\left(\widehat{\tau^{\prime}+\tau}\right)-\mathbf{z}\right) \cdot \dot{\boldsymbol{\gamma}}_{T}\left(\tau^{\prime}+\tau\right) \\
& \left.+\left(\boldsymbol{\gamma}_{R}\left(\widehat{\tau^{\prime}+\tau}\right)-\mathbf{z}\right) \cdot \dot{\boldsymbol{\gamma}}_{R}\left(\tau^{\prime}+\tau\right)\right] .
\end{aligned}
$$

Note that $f_{d}$ is the bistatic Doppler frequency induced by the radial movements of the transmitter and receiver with respect to the scatterer $z$ on the ground. Unless stated otherwise, we use frequency in hertz for the rest of this paper.

We write the forward model for bistatic DSAR as follows:

$$
\begin{aligned}
d\left(\tau^{\prime}, \tau, \mu\right) \approx & \mathcal{F}[\rho]\left(\tau^{\prime}, \tau, \mu\right)=\int \mathrm{e}^{-\mathrm{i} \varphi\left(t, \boldsymbol{z}, \tau^{\prime}, \tau, \mu\right)} \\
& \times A\left(\boldsymbol{z}, t, \tau^{\prime}, \tau, \mu\right) \rho(\boldsymbol{z}) d t d \boldsymbol{z}
\end{aligned}
$$

where

$$
\varphi\left(t, \boldsymbol{z}, \tau^{\prime}, \tau, \mu\right)=2 \pi t\left[f_{d}\left(\tau^{\prime}, \tau, \mathbf{z}\right)-f_{0}(1-\mu)\right]
$$

and

$$
\begin{aligned}
A\left(\boldsymbol{z}, t, \tau^{\prime}, \tau, \mu\right)= & \mathrm{e}^{\mathrm{i} \omega_{0}\left[(1-\mu) \tau^{\prime}+\tau-R_{\mathrm{TR}}\left(\tau^{\prime}+\tau, \boldsymbol{z}\right) / c_{0}\right]} \\
& \times A_{\tilde{p}}\left(\mathbf{z}, t, \tau^{\prime}, \tau, \mu\right) \\
& \times A_{\mathrm{TR}}\left(\omega_{0}, \mathbf{z}, t, \tau^{\prime}, \tau\right) \phi(t) .
\end{aligned}
$$

We refer to $\mathcal{F}$ as the forward modeling operator, and $\varphi$ and $A$ as the phase and amplitude terms of the linear operator $\mathcal{F}$, respectively. For the monostatic forward model, see [8].

We assume that, for some $m_{A}, A$ satisfies the inequality

$$
\begin{aligned}
\sup _{(t, \mu, \tau, \boldsymbol{z}) \in \mathcal{U}}\left|\partial_{t}^{\alpha_{t}} \partial_{\mu}^{\alpha_{\mu}} \partial_{\tau}^{\beta} \partial_{z_{1}}^{\epsilon_{1}} \partial_{z_{2}}^{\epsilon_{2}} A\left(\boldsymbol{z}, t, \tau^{\prime}, \tau, \mu\right)\right| \\
\leq C_{A}\left(1+t^{2}\right)^{\frac{\left(m_{A}-\left|\alpha_{t}\right|\right)}{2}}
\end{aligned}
$$

where $\mathcal{U}$ is any compact subset of $\mathbb{R} \times \mathbb{R}^{+} \times \mathbb{R} \times \mathbb{R}^{2}$, and the constant $C_{A}$ depends on $\mathcal{U}, \alpha_{t, \mu}, \beta, \epsilon_{1,2}$. This assumption is needed in order to make various stationary phase calculations hold. In practice, (22) is satisfied when the antennas are sufficiently away from the illuminated scene.

Under the assumption (22), (19) defines $\mathcal{F}$ as a Fourier integral operator [20].

\section{Critical Points of the Bistatic DSAR Forward Model}

The leading order contribution of $\mathcal{F}$ comes from those points lying in the critical set of the phase, i.e., the intersection of the illuminated topography $(\boldsymbol{z}, \psi(\boldsymbol{z}))$ and the surface $\{\mathbf{z} \in$ $\left.\mathbb{R}^{3}: f_{d}\left(\tau^{\prime}, \tau, \mathbf{z}\right)=f_{0}(1-\mu)\right\}$. We refer to this surface, on which the points $\mathbf{z} \in \mathbb{R}^{3}$ have the same bistatic Doppler frequency, as the bistatic iso-Doppler surface. Note that the shape of the bistatic iso-Doppler surface depends on the flight trajectories of the transmitter and receiver and is not necessarily a cone, which happens only when the transmitter and receiver are colocated.

We denote the curves formed by the intersection of the bistatic iso-Doppler surface and ground topography by

$$
F\left(\tau^{\prime}, \tau, \mu\right)=\left\{z: f_{d}\left(\tau^{\prime}, \tau, \mathbf{z}\right)=f_{0}(1-\mu) \cap \psi(z)=z_{3}\right\}
$$

and refer to $F\left(\tau^{\prime}, \tau, \mu\right)$ as the bistatic iso-Doppler contour associated with $\left(\tau^{\prime}, \tau, \mu\right)$. The points that lie on these isoDoppler contours are the leading-order contributions to the forward model given in (19). Fig. 1 shows the bistatic isoDoppler contours for different flight trajectories over a flat topography.

High-frequency analysis of the forward model $\mathcal{F}$ shows that the windowed, scaled, and translated correlations between the received signal and the transmitted signal are the projections of the scene reflectivity onto the bistatic iso-Doppler contours.

\section{IMAGE FORMATION}

Our objective is to reconstruct $\rho(\boldsymbol{z})$ based on the forward model given in (19). Since $\mathcal{F}$ is a Fourier integral operator, we form the image of $\rho(\boldsymbol{z})$ by a suitable FBP of the data $d\left(\tau^{\prime}, \tau, \mu\right)$ onto $F\left(\tau^{\prime}, \tau, \mu\right)$ for a fixed $\tau^{\prime}$ and coherently superimposing over all $\tau^{\prime}$.

In this section, we first define the FBP operator used for image reconstruction. Then, we present the PSF of the FBP operator and analyze its high-frequency behavior to determine the location and orientation of the singularities of the scene that are reconstructed as well as the resolution of the reconstructed images. We next discuss the design of the FBP filter. We present a detailed resolution analysis and the algorithmic implementation, along with a computational complexity analysis of our imaging method in Sections III-E and III-F.

\section{A. Filtered Backprojection Operator}

We form an image of the scene by the superposition of the filtered and backprojected data as follows:

$$
\tilde{\rho}(\boldsymbol{z}):=\int \mathcal{K}_{\tau^{\prime}}[d]\left(z, \tau^{\prime}\right) d \tau^{\prime}
$$

where

$$
\begin{aligned}
\mathcal{K}_{\tau^{\prime}}[d]\left(\boldsymbol{z}, \tau^{\prime}\right)= & \int \mathrm{e}^{\mathrm{i} \varphi\left(t, \boldsymbol{z}, \tau^{\prime}, \tau, \mu\right)} Q\left(\boldsymbol{z}, t, \tau^{\prime}, \tau\right) \\
& \times d\left(\tau^{\prime}, \tau, \mu\right) d t d \tau d \mu
\end{aligned}
$$

is the FBP operator with respect to a fixed $\tau^{\prime} . Q$ in (25) is the filter to be determined below.

We assume that for some $m_{Q}, Q$ satisfies the inequality

$$
\begin{aligned}
\sup _{\left(t, \tau^{\prime}, \tau, \boldsymbol{z}\right) \in \mathcal{U}}\left|\partial_{t}^{\alpha_{t}} \partial_{\tau^{\prime}}^{\beta_{1}} \partial_{\tau}^{\beta_{2}} \partial_{z_{1}}^{\epsilon_{1}} \partial_{z_{2}}^{\epsilon_{2}} Q\left(\boldsymbol{z}, t, \tau^{\prime}, \tau\right)\right| \\
\leq C_{Q}\left(1+t^{2}\right)^{\frac{\left(m_{Q}-\left|\alpha_{t}\right|\right)}{2}}
\end{aligned}
$$

where $\mathcal{U}$ is any compact subset of $\mathbb{R} \times \mathbb{R} \times \mathbb{R} \times \mathbb{R}^{2}$, and the constant $C_{Q}$ depends on $\mathcal{U}, \alpha_{t}, \beta_{1,2}$, and $\epsilon_{1,2}$. Assumption (22) makes $\mathcal{K}_{\tau^{\prime}}$ a Fourier integral operator. 


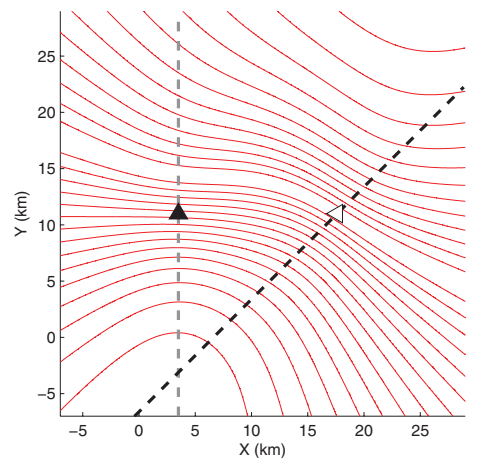

(a)

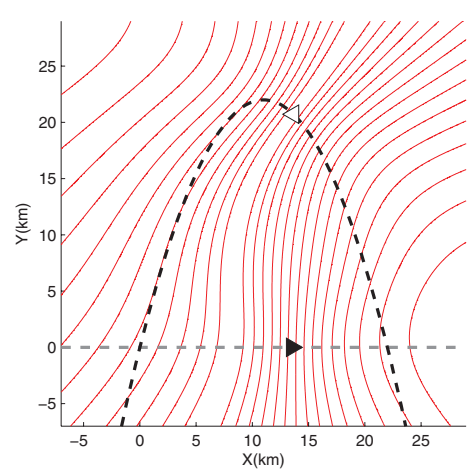

(b)

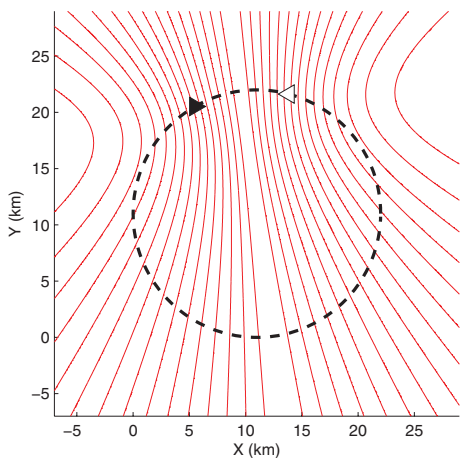

(c)

Fig. 1. Bistatic iso-Doppler contours $F\left(\tau^{\prime}, \tau, \mu\right)$ determined for a certain $\tau^{\prime}$ and $\tau$ for three different transmitter and receiver flight trajectories as indicated by the gray and black dashed lines, respectively. The black and white triangles denote the corresponding positions of the transmitter and receiver. (a) Transmitter and receiver are both traversing straight linear flight trajectories. $\gamma_{T}(s)=[3.5, s, 6.5] \mathrm{km}$ and $\boldsymbol{\gamma}_{R}(s)=[(s-7), s, 6.5] \mathrm{km}$ where $s=v t$ with speed $v=261 \mathrm{~m} / \mathrm{s}$. (b) Transmitter is traversing a straight linear flight trajectory, $\gamma_{R}(s)=[s, 0,6.5] \mathrm{km}$ and the receiver is traversing a parabolic flight trajectory, $\boldsymbol{\gamma}_{T}(s)=\left[s,(s-11)^{2} * 22 / 121,6.5\right] \mathrm{km}$. (c) Transmitter and receiver are both traversing a circular flight trajectory. $\boldsymbol{\gamma}_{T}(s)=\boldsymbol{\gamma}_{C}(s)$ and $\boldsymbol{\gamma}_{R}(s)=\boldsymbol{\gamma}_{C}(s-\pi / 4)$ where $\gamma_{C}(s)=[11+11 \cos s, 11+11 \sin s, 6.5] \mathrm{km}$ with $s=(v / R) t$ where speed $v=261 \mathrm{~m} / \mathrm{s}$ and radius $R=11 \mathrm{~km}$. Note that each red curve corresponds to a distinct value of $\mu$.

\section{B. PSF of the Imaging Operator}

We rewrite $\tilde{\rho}(\boldsymbol{z})$ as

$$
\tilde{\rho}(\boldsymbol{z})=\int \mathcal{K}_{\tau^{\prime}} \mathcal{F}[\rho](\boldsymbol{z}) d \tau^{\prime}=\int L\left(\boldsymbol{z}, \boldsymbol{z}^{\prime}\right) \rho\left(\boldsymbol{z}^{\prime}\right) d \boldsymbol{z}^{\prime}
$$

where $L\left(z, z^{\prime}\right)$ is the PSF of the imaging operator given by

$$
L\left(\boldsymbol{z}, \boldsymbol{z}^{\prime}\right)=\int L\left(\boldsymbol{z}, \boldsymbol{z}^{\prime}, \tau^{\prime}\right) d \tau^{\prime}
$$

and

$$
\begin{aligned}
L\left(\boldsymbol{z}, \boldsymbol{z}^{\prime}, \tau^{\prime}\right)= & \int \mathrm{e}^{\mathrm{i}\left[\varphi\left(t, \boldsymbol{z}, \tau^{\prime}, \tau, \mu\right)-\varphi\left(t^{\prime}, \boldsymbol{z}^{\prime}, \tau^{\prime}, \tau, \mu\right)\right]} Q\left(\boldsymbol{z}, t, \tau^{\prime}, \tau\right) \\
& \times A\left(\boldsymbol{z}^{\prime}, t^{\prime}, \tau^{\prime}, \tau, \mu\right) d t d t^{\prime} d \tau d \mu .
\end{aligned}
$$

Note that $L\left(z, \boldsymbol{z}^{\prime}, \tau^{\prime}\right)$ can be viewed as the PSF of the partial imaging operator $\mathcal{K}_{\tau^{\prime}}$.

We define

$$
\Phi_{K}:=\varphi\left(t, \boldsymbol{z}, \tau^{\prime}, \tau, \mu\right)-\varphi\left(t^{\prime}, \boldsymbol{z}^{\prime}, \tau^{\prime}, \tau, \mu\right)
$$

as the phase of $\mathcal{K}_{\tau^{\prime}} \mathcal{F}$ and use the stationary phase theorem [21]-[23] to approximate the $t^{\prime}$ and $\mu$ integrations in (29). We compute

$$
\partial_{t^{\prime}} \Phi_{K}=-2 \pi\left[f_{d}\left(\tau^{\prime}, \tau, \boldsymbol{z}^{\prime}\right)-f_{0}(1-\mu)\right]
$$

and

$$
\partial_{\mu} \Phi_{K}=2 \pi f_{0}\left(t-t^{\prime}\right) .
$$

The stationary points of the phase satisfying $\partial_{t^{\prime}, \mu} \Phi_{K}=0$ imply that ${ }^{2}$

$$
\mu=1-f_{d}\left(\tau^{\prime}, \tau, \boldsymbol{z}^{\prime}\right) / f_{0}
$$

and $t=t^{\prime}$.

\footnotetext{
${ }^{2}$ The determinant of the Hessian matrix of $\Phi_{K}$ is $(2 \pi)^{2} f_{0}^{2}$. Thus, the stationary points are nondegenerate.
}

Substituting the results back into (29), we obtain

$$
\begin{aligned}
L\left(\boldsymbol{z}, \boldsymbol{z}^{\prime}, \tau^{\prime}\right)= & \int \mathrm{e}^{\mathrm{i} 2 \pi t\left[f_{d}\left(\tau^{\prime}, \tau, \boldsymbol{z}\right)-f_{d}\left(\tau^{\prime}, \tau, \boldsymbol{z}^{\prime}\right)\right]} Q\left(\boldsymbol{z}, t, \tau^{\prime}, \tau\right) \\
& \times A\left(\boldsymbol{z}^{\prime}, t, \tau^{\prime}, \tau, 1-f_{d}\left(\tau^{\prime}, \tau, \boldsymbol{z}^{\prime}\right) / f_{0}\right) d t d \tau .
\end{aligned}
$$

To simplify our notation, for the rest of our paper, we let

$$
A\left(\boldsymbol{z}^{\prime}, t, \tau^{\prime}, \tau\right)=A\left(\boldsymbol{z}^{\prime}, t, \tau^{\prime}, \tau, 1-f_{d}\left(\tau^{\prime}, \tau, \boldsymbol{z}^{\prime}\right) / f_{0}\right) .
$$

\section{Critical Points of the Image Fidelity Operator $\mathcal{K}_{\tau^{\prime}} \mathcal{F}$}

The main contributions of $\mathcal{K}_{\tau^{\prime}} \mathcal{F}$ come from the critical points of its phase that satisfy the conditions [24]

$$
\begin{gathered}
\partial_{t}\left(2 \pi t\left[f_{d}\left(\tau^{\prime}, \tau, \boldsymbol{z}\right)-f_{d}\left(\tau^{\prime}, \tau, \boldsymbol{z}^{\prime}\right)\right]\right)=0 \Rightarrow f_{d}\left(\tau^{\prime}, \tau, \boldsymbol{z}\right) \\
=f_{d}\left(\tau^{\prime}, \tau, \boldsymbol{z}^{\prime}\right)
\end{gathered}
$$

and

$$
\begin{gathered}
\partial_{\tau}\left(2 \pi t\left[f_{d}\left(\tau^{\prime}, \tau, \boldsymbol{z}\right)-f_{d}\left(\tau^{\prime}, \tau, \boldsymbol{z}^{\prime}\right)\right]\right)=0 \Rightarrow \dot{f}_{d}\left(\tau^{\prime}, \tau, \boldsymbol{z}\right) \\
=\dot{f}_{d}\left(\tau^{\prime}, \tau, \boldsymbol{z}^{\prime}\right)
\end{gathered}
$$

where $\dot{f}_{d}$ denotes the first-order partial derivative of $f_{d}$ with respect to $\tau$, i.e., $\dot{f}_{d}=\partial f_{d} / \partial \tau$. We refer to $\dot{f}_{d}\left(\tau^{\prime}, \tau, \mathbf{z}\right)$ as the bistatic Doppler rate.

Using (18), we obtain

$$
\begin{aligned}
\dot{f}_{d}\left(\tau^{\prime}, \tau, \boldsymbol{z}\right)=\frac{f_{0}}{c_{0}}[ & \frac{1}{\left|\boldsymbol{\gamma}_{T}\left(\tau^{\prime}+\tau\right)-\mathbf{z}\right|}\left|\dot{\boldsymbol{\gamma}}_{T, \perp}\left(\tau^{\prime}+\tau, \mathbf{z}\right)\right|^{2} \\
& +\left(\boldsymbol{\gamma}_{T}\left(\widehat{\tau^{\prime}+\tau}\right)-\mathbf{z}\right) \cdot \ddot{\boldsymbol{\gamma}}_{T}\left(\tau^{\prime}+\tau\right) \\
& +\frac{1}{\left|\boldsymbol{\gamma}_{R}\left(\tau^{\prime}+\tau\right)-\mathbf{z}\right|}\left|\dot{\boldsymbol{\gamma}}_{R, \perp}\left(\tau^{\prime}+\tau, \mathbf{z}\right)\right|^{2} \\
& \left.+\left(\boldsymbol{\gamma}_{R}\left(\widehat{\tau^{\prime}+\tau}\right)-\mathbf{z}\right) \cdot \ddot{\boldsymbol{\gamma}}_{R}\left(\tau^{\prime}+\tau\right)\right]
\end{aligned}
$$

where

$$
\begin{aligned}
\dot{\boldsymbol{\gamma}}_{T(R), \perp}\left(\tau^{\prime}+\tau, \mathbf{z}\right)= & \left.\dot{\boldsymbol{\gamma}}_{T(R)}\left(\tau^{\prime}+\tau\right)-\left(\boldsymbol{\gamma}_{T(R)} \widehat{\left(\tau^{\prime}+\tau\right.}\right)-\mathbf{z}\right) \\
& \times\left[\left(\boldsymbol{\gamma}_{T(R)} \widehat{\left(\tau^{\prime}+\tau\right)}-\mathbf{z}\right) \cdot \dot{\boldsymbol{\gamma}}_{T(R)}\left(\tau^{\prime}+\tau\right)\right] .
\end{aligned}
$$




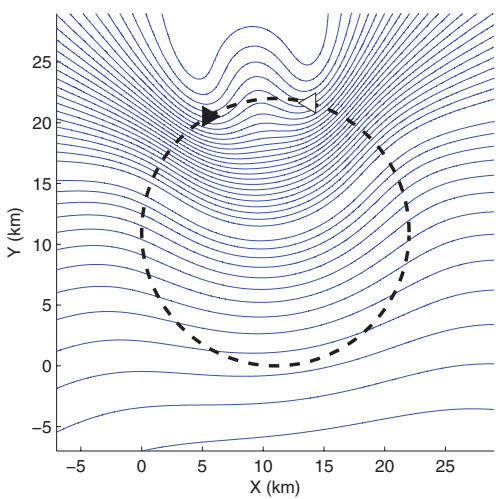

(a)

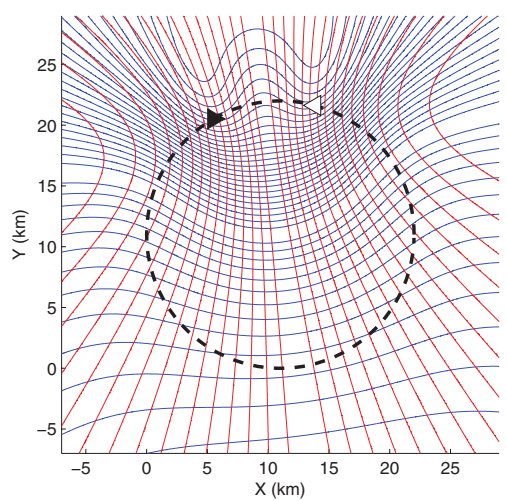

(b)

Fig. 2. (a) Bistatic iso-Doppler rate contours $\dot{F}\left(\tau^{\prime}, \tau, C\right)$ for the Doppler rate $\dot{f}_{d}(22.0674 \mathrm{~s}, 66.2021 \mathrm{~s}, \mathbf{z})$. (b) Illustration of the intersection of the bistatic iso-Doppler contours for the bistatic Doppler $f_{d}(22.0674 \mathrm{~s}, 66.2021 \mathrm{~s}, \mathbf{z})$, and bistatic iso-Doppler rate contours for the bistatic Doppler rate $\dot{f}_{d}(22.0674 \mathrm{~s}, 66.2021 \mathrm{~s}, \mathbf{z})$. The transmitter and receiver are traversing a circular flight trajectory (indicated by the dashed line) over a flat topography. $\gamma_{T}(s)=\gamma_{C}(s)$ and $\gamma_{R}(s)=\gamma_{C}(s-\pi / 4)$, where black and white triangles denote the position of the transmitter and receiver at $s=\pi / 6$, corresponding to $\tau^{\prime}=22.0674 \mathrm{~s}$, respectively. $\gamma_{C}(s)=[11+11 \cos s, 11+11 \sin s, 6.5] \mathrm{km}$ with $s=(v / R) \tau$ where speed $v=261 \mathrm{~m} / \mathrm{s}$ and radius $R=11 \mathrm{~km}$.

denotes the projection of the platform velocity $\dot{\gamma}_{T(R)}\left(\tau^{\prime}+\tau\right)$ onto the plane whose normal direction is along $\boldsymbol{\gamma}_{T(R)} \widehat{\left(\tau^{\prime}+\tau\right)}-\mathbf{z}$. Note that the summation of the first two terms in the square bracket in (38) denotes the relative radial acceleration of the transmitter in the direction of $\boldsymbol{\gamma}_{T}\left(\widehat{\tau^{\prime}+\tau}\right)-\mathbf{z}$ evaluated at $\tau^{\prime}+\tau$ with respect to the scatterer $z$ on the ground, while the summation of the last two terms in the square bracket corresponds to the relative radial acceleration of the receiver with respect to the scatterer $\boldsymbol{z}$.

We refer to the locus of points formed by the intersection of the illuminated surface and $\left\{\mathbf{z} \in \mathbb{R}^{3}: \dot{f}_{d}\left(\tau^{\prime}, \tau, \mathbf{z}\right)=C\right\}$, for some constant $C$, as the iso-Doppler rate contour and denote it by

$$
\dot{F}\left(\tau^{\prime}, \tau, C\right)=\left\{z: \dot{f}_{d}\left(\tau^{\prime}, \tau, \mathbf{z}\right)=C \cap \boldsymbol{\psi}(z)=z_{3}\right\} .
$$

Fig. 2(a) shows the bistatic iso-Doppler rate contours with a fixed $\tau^{\prime}+\tau$ for a circular flight trajectory and flat topography.

The critical points $z$ of the phase of the image fidelity operator, i.e., $\mathcal{K}_{\tau^{\prime}} \mathcal{F}$, are those points that lie on the intersection of the bistatic iso-Doppler curves $F\left(\tau^{\prime}, \tau, \mu\right)$ and bistatic isoDoppler rate curves $\dot{F}\left(\tau^{\prime}, \tau, C\right)$, as shown in Fig. 2(b). We assume that the flight trajectory of the radar is smooth and that the antenna beam pattern is focused on a region of interest where each pair of bistatic iso-Doppler $F\left(\tau^{\prime}, \tau, \mu\right)$ and isoDoppler rate $\dot{F}\left(\tau^{\prime}, \tau, C\right)$ contours intersect at a single point within the region of interest. In other words, we assume that the only critical point within the region of interest is $\boldsymbol{z}=\boldsymbol{z}^{\prime}$.

The analysis of the PSF of the imaging operator above shows that the backprojection operator reconstructs the visible edges of the scene at the intersection of the bistatic iso-Doppler and bistatic iso-Doppler rate contours. It also shows that the imaging method uses not only the Doppler induced by the velocity of the antennas but also the acceleration of antennas in certain directions to synthesize an aperture.

\section{Determination of the FBP Filter}

The filter $Q$ can be determined with respect to various criteria [25]. We will determine $Q$ so that the PSF of the imaging operator $L\left(z, z^{\prime}, \tau^{\prime}\right)$ is as close as possible to the Dirac-delta function $\delta\left(\boldsymbol{z}-\boldsymbol{z}^{\prime}\right)$. This choice of $Q$ ensures that $\mathcal{K}_{\tau^{\prime}}$ reconstructs the visible edges of the scene not only at the correct location with correct orientation but also with the correct strength [19], [25]-[27].

To determine the filter, we linearize $f_{d}\left(\tau^{\prime}, \tau, z^{\prime}\right)$ around $z^{\prime}=z$ using the fundamental theorem of calculus [28] and approximate

$$
f_{d}\left(\tau^{\prime}, \tau, \boldsymbol{z}\right)-f_{d}\left(\tau^{\prime}, \tau, \boldsymbol{z}^{\prime}\right) \approx\left(\boldsymbol{z}-\boldsymbol{z}^{\prime}\right) \cdot \nabla_{\boldsymbol{z}} f_{d}\left(\tau^{\prime}, \tau, \boldsymbol{z}\right)
$$

and write

$$
A\left(z^{\prime}, t, \tau^{\prime}, \tau\right) \approx A\left(z, t, \tau^{\prime}, \tau\right)
$$

Thus, (34) becomes

$$
\begin{aligned}
L\left(\boldsymbol{z}, \boldsymbol{z}^{\prime}, \tau^{\prime}\right)= & \int \mathrm{e}^{\mathrm{i} t\left(\boldsymbol{z}-\boldsymbol{z}^{\prime}\right) \cdot \boldsymbol{\Xi}\left(\tau^{\prime}, \tau, \boldsymbol{z}\right)} \\
& \times Q\left(\boldsymbol{z}, t, \tau^{\prime}, \tau\right) A\left(\boldsymbol{z}, t, \tau^{\prime}, \tau\right) d t d \tau
\end{aligned}
$$

where

$$
\boldsymbol{\Xi}\left(\tau^{\prime}, \tau, \boldsymbol{z}\right)=2 \pi \nabla_{\boldsymbol{z}} f_{d}\left(\tau^{\prime}, \tau, \boldsymbol{z}\right)
$$

For each $\tau^{\prime}$ and $\boldsymbol{z}$, we make the following change of variables:

$$
(t, \tau) \rightarrow \boldsymbol{\xi}=t \boldsymbol{\Xi}\left(\tau^{\prime}, \tau, \boldsymbol{z}\right)
$$

and write (43) as follows:

$$
\begin{aligned}
L\left(\boldsymbol{z}, \boldsymbol{z}^{\prime}, \tau^{\prime}\right)= & \int_{\Omega_{\tau^{\prime}, \boldsymbol{z}}} \mathrm{e}^{\mathrm{i}\left(\boldsymbol{z}-\boldsymbol{z}^{\prime}\right) \cdot \boldsymbol{\xi}} \\
& \times Q\left(\boldsymbol{z}, \tau^{\prime}, \boldsymbol{\xi}\right) A\left(\boldsymbol{z}, \tau^{\prime}, \boldsymbol{\xi}\right) \eta\left(\boldsymbol{z}, \tau^{\prime}, \boldsymbol{\xi}\right) d \boldsymbol{\xi}
\end{aligned}
$$

where

$$
\begin{aligned}
& Q\left(z, \tau^{\prime}, \xi\right)=Q\left(z, t(\xi), \tau^{\prime}, \tau(\xi)\right) \\
& A\left(z, \tau^{\prime}, \xi\right)=A\left(z, t(\xi), \tau^{\prime}, \tau(\xi)\right)
\end{aligned}
$$

and

$$
\eta\left(\boldsymbol{z}, \tau^{\prime}, \boldsymbol{\xi}\right)=\left|\frac{\partial(t, \tau)}{\partial \boldsymbol{\xi}}\right|=|t|^{-1}\left|\operatorname{det}\left[\begin{array}{c}
\boldsymbol{\Xi}\left(\tau^{\prime}, \tau, \boldsymbol{z}\right) \\
\partial_{\tau} \boldsymbol{\Xi}\left(\tau^{\prime}, \tau, \boldsymbol{z}\right)
\end{array}\right]\right|^{-1}
$$




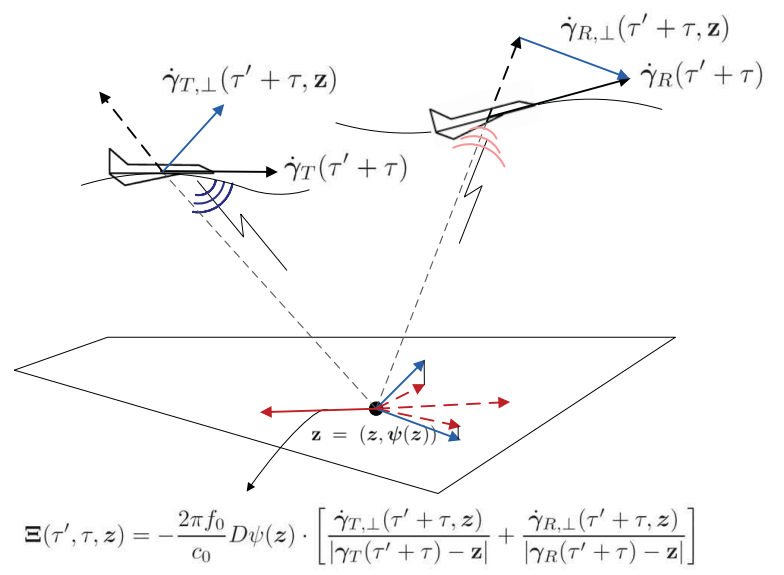

Fig. 3. Illustration of the vector $\boldsymbol{\Xi}\left(\tau^{\prime}, \tau, \boldsymbol{z}\right)$ in the data collection manifold $\Omega_{\tau^{\prime}, \boldsymbol{z}}$ for flat topography, $\psi(\boldsymbol{z})=0$.

is the determinant of the Jacobian that comes from the change of variables given in (45). Note that the notation det in (48) denotes the matrix determinant. The domain of integration in (46) is given by

$\Omega_{\tau^{\prime}, \boldsymbol{z}}=\left\{\boldsymbol{\xi}=t \boldsymbol{\Xi}\left(\tau^{\prime}, \tau, \boldsymbol{z}\right) \mid A\left(\boldsymbol{z}, t, \tau^{\prime}, \tau\right) \neq 0, \quad t, \tau, \tau^{\prime} \in \mathbb{R}\right\}$.

We refer to $\Omega_{\tau^{\prime}, \boldsymbol{z}}$ as the partial data collection manifold at $\tau^{\prime}, \boldsymbol{z}$ obtained for a fixed $\tau^{\prime}$, and refer to the union $\bigcup_{\tau^{\prime}} \Omega_{\tau^{\prime}, \boldsymbol{z}}$ as the data collection manifold at $\boldsymbol{z}$ and denote it by $\boldsymbol{\Omega}_{\boldsymbol{z}}$. This set determines many of the properties of the reconstructed image.

Using (44) and (18), we obtain

$$
\begin{aligned}
\mathbf{\Xi}\left(\tau^{\prime}, \tau, \boldsymbol{z}\right)= & -\frac{2 \pi f_{0}}{c_{0}} D \psi(\boldsymbol{z}) \\
& \cdot\left[\frac{\dot{\boldsymbol{\gamma}}_{T, \perp}\left(\tau^{\prime}+\tau, \boldsymbol{z}\right)}{\left|\boldsymbol{\gamma}_{T}\left(\tau^{\prime}+\tau\right)-\mathbf{z}\right|}+\frac{\dot{\boldsymbol{\gamma}}_{R, \perp}\left(\tau^{\prime}+\tau, \boldsymbol{z}\right)}{\left|\boldsymbol{\gamma}_{R}\left(\tau^{\prime}+\tau\right)-\mathbf{z}\right|}\right]
\end{aligned}
$$

where

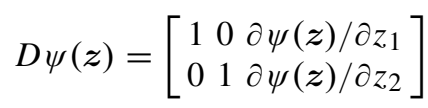

and $\dot{\boldsymbol{\gamma}}_{T(R), \perp}\left(\tau^{\prime}+\tau, \boldsymbol{z}\right)$ is the projection of $\dot{\gamma}_{T(R)}\left(\tau^{\prime}+\tau\right)$ onto the plane whose normal is $\boldsymbol{\gamma}_{T(R)} \widehat{\left(\tau^{\prime}+\tau\right)}-\mathbf{z}$ as defined by (39).

We present a graphical illustration of $\boldsymbol{\Xi}\left(\tau^{\prime}, \tau, \boldsymbol{z}\right)$ in Fig. 3 for flat topography. From (45) and (46), we see that $t \boldsymbol{\Xi}\left(\tau^{\prime}, \tau, \boldsymbol{z}\right)$ is the Fourier vector that contributes to the reconstruction of the image at pixel $\mathbf{z} \in \mathbb{R}^{3}$ for fixed $\tau$ and $\tau^{\prime}$.

To approximate the PSF $L\left(\boldsymbol{z}, \boldsymbol{z}^{\prime}, \tau^{\prime}\right)$ in (46) with the Diracdelta function, we choose the filter as follows:

$$
Q\left(z, t, \tau^{\prime}, \tau\right)=\frac{\chi \Omega_{\tau^{\prime}, z}}{\eta\left(z, \tau^{\prime}, \xi\right)} \frac{A^{*}\left(z, t, \tau^{\prime}, \tau\right)}{\left|A\left(z, t, \tau^{\prime}, \tau\right)\right|^{2}}
$$

where $\chi_{\Omega_{\tau^{\prime}, z}}$ is a smooth cut-off function that prevents division by zero in (52).

Note that irrespective of the choice of the filter, the backprojection operator recovers the visible edges of the scene at the correct location and orientation. With the choice of filter given in (52), assuming that (26) is satisfied, the resulting FBP operator can recover not only the correct location and orientation but also the correct strength of the visible edges [25], [26], [29], [30].

\section{E. Resolution Analysis for Bistatic DSAR}

Substituting (52) into (43) and the result back into (24), we obtain

$$
\begin{aligned}
\tilde{\rho}(\boldsymbol{z}) & :=\int \mathcal{K}_{\tau^{\prime}} \mathcal{F}[\rho](\boldsymbol{z}) d \tau^{\prime} \\
& =\int_{\Omega_{\tau^{\prime}, \boldsymbol{z}}} \mathrm{e}^{\mathrm{i}\left(\boldsymbol{z}-\boldsymbol{z}^{\prime}\right) \cdot \boldsymbol{\xi}} \rho\left(\boldsymbol{z}^{\prime}\right) d \boldsymbol{z}^{\prime} d \boldsymbol{\xi} d \tau^{\prime} .
\end{aligned}
$$

Equation (53) shows that the image $\tilde{\rho}$ is a band-limited version of $\rho$ whose bandwidth is determined by the data collection manifold $\Omega_{\boldsymbol{z}}$. The larger the data collection manifold, the better the resolution of the reconstructed image.

Microlocal analysis of (53) shows that an edge at point $z$ is visible if the direction $\boldsymbol{n}_{\boldsymbol{z}}$ normal to the edge is contained in the data collection manifold $\Omega_{\boldsymbol{z}}$ [19], [25]-[27]. Consequently, an edge at point $\boldsymbol{z}$ with normal $\boldsymbol{n}_{\boldsymbol{z}}$ is visible if there exists $\tau^{\prime}, \tau$ such that $\boldsymbol{\xi}$ is parallel to $\boldsymbol{n}_{\boldsymbol{z}}$. Furthermore, as indicated by (49) and (44), the bandwidth contribution of $\xi$ to the image at $z$ is given by

$$
\frac{2 \pi f_{0}}{c_{0}} L_{\phi}\left|D \psi(\boldsymbol{z}) \cdot\left[\frac{\dot{\boldsymbol{\gamma}}_{T, \perp}\left(\tau^{\prime}+\tau, \boldsymbol{z}\right)}{\left|\boldsymbol{\gamma}_{T}\left(\tau^{\prime}+\tau\right)-\mathbf{z}\right|}+\frac{\dot{\boldsymbol{\gamma}}_{R, \perp}\left(\tau^{\prime}+\tau, \boldsymbol{z}\right)}{\left|\boldsymbol{\gamma}_{R}\left(\tau^{\prime}+\tau\right)-\mathbf{z}\right|}\right]\right|
$$

where $L_{\phi}$ denotes the length of the support of $\phi(t)$.

Equation (54) shows that, as the carrier frequency of the transmitted signal $f_{0}$ becomes higher, the magnitude of $\boldsymbol{\xi}$ gets larger, which results in a higher resolution image. The sharpness of the reconstructed edges is also directly proportional to the length of the support of $\phi(t)$. Furthermore, (54) shows that the resolution depends on the range of the antenna to the scene via the terms $\left|\boldsymbol{\gamma}_{T}\left(\tau^{\prime}+\tau\right)-\mathbf{z}\right|$ and $\left|\boldsymbol{\gamma}_{R}\left(\tau^{\prime}+\tau\right)-\mathbf{z}\right|$, as well as the speed of the transmitter and receiver via the terms $\dot{\gamma}_{T, \perp}$ and $\dot{\gamma}_{R, \perp}$. As the antennas move away from the scene, or the speed of the transmitter or the receiver decreases in certain directions, the magnitude of $\boldsymbol{\xi}$ decreases, which results in reduced resolution. Additionally, the increase in the number of $\tau$ samples and the time windows (indicated by $\tau^{\prime}$ ) used for imaging also lead to a larger data collection manifold, and hence improved resolution. We summarize the parameters that affect the resolution of the reconstructed image in Table I. Note that, in practice, one needs take into account the interaction of the parameters in Table I with the assumptions of the imaging model. For example, an arbitrarily long time window $L_{\phi}$ or relatively short antenna-to-scene distance is inconsistent with the approximations made in (15) and (16).

We also note that in a scenario where the transmitter and the receiver are flying along the same straight trajectory, the scatterers in the forward-looking and backward-looking region of the radar platforms cannot be reconstructed since both $\dot{\gamma}_{T, \perp}$ and $\dot{\gamma}_{R, \perp}$ will be zero in that region. Furthermore, given the velocities of the radar platforms and the look directions of the 


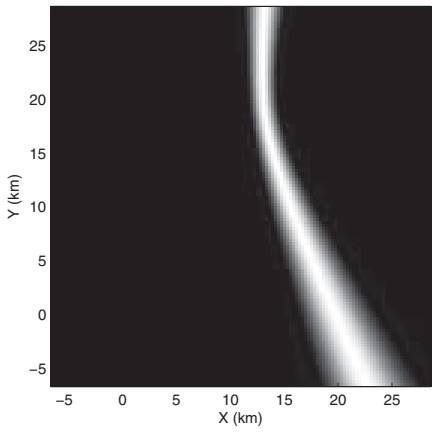

(a)

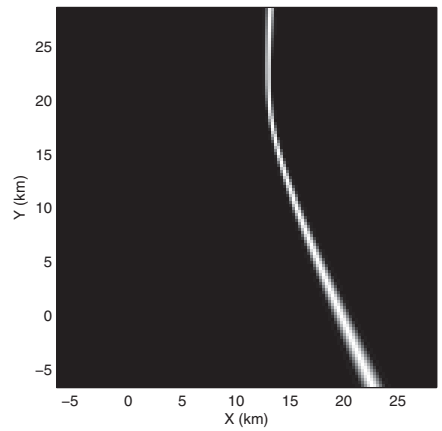

(b)

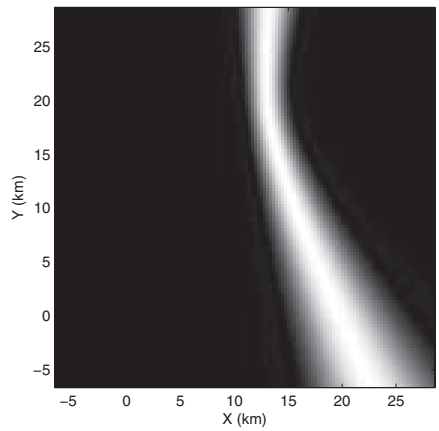

(c)

Fig. 4. Bistatic iso-Doppler contours obtained by backprojecting the data for fixed $\tau$ and $\tau^{\prime}$ with (a) $L_{\phi}=0.0107 \mathrm{~s}, f_{0}=800 \mathrm{MHz}$, (b) $L_{\phi}=0.0427 \mathrm{~s}$, $f_{0}=800 \mathrm{MHz}$, and (c) $L_{\phi}=0.0107 \mathrm{~s}, f_{0}=500 \mathrm{MHz}$. The received signal is generated using (7) for a point target located at [15.125, 11,0] km. The location of the transmitter and receiver are the same as in Fig. 1. A single-frequency continuous transmitted waveform is used.

TABLE I

Parameters That AfFect the Bistatic DSAR Image Resolution

\begin{tabular}{lcc}
\hline \hline Parameter & Increase( $\uparrow)$ & Resolution \\
\hline Carrier frequency: $f_{0}$ & $\uparrow$ & $\uparrow$ \\
Length of the windows $L_{\phi}$ & $\uparrow$ & $\uparrow$ \\
Distance $\left|\boldsymbol{\gamma}_{T}-\mathbf{z}\right|,\left|\boldsymbol{\gamma}_{R}-\mathbf{z}\right|$ & $\uparrow$ & $\downarrow$ \\
Antenna velocity $\dot{\boldsymbol{\gamma}}_{T}$ or $\dot{\boldsymbol{\gamma}}_{R}$ & $\uparrow$ & $\uparrow$ \\
Number of $\tau$ samples & $\uparrow$ & $\uparrow$ \\
Number of time windows & $\uparrow$ & $\uparrow$ \\
\hline \hline
\end{tabular}

Higher $(\uparrow)$ or Lower $(\downarrow)$.

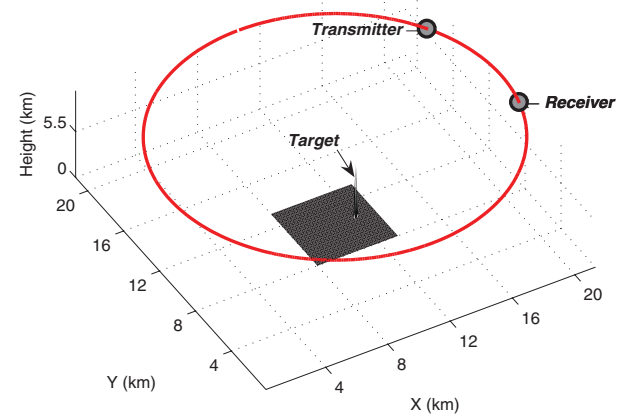

Fig. 5. 3-D schematic of the simulation setup. The dark region denotes the scene considered in the simulations. The transmitter/receiver antennas traverse a continuum of positions along the circular trajectory as shown by the red dashed line. At a certain time instant, the transmitter and receiver are located at the positions indicated by the solid dots.

transmitter and receiver, $\dot{\gamma}_{T, \perp}$ may become equal to $-\dot{\gamma}_{R, \perp}$ for some scatters, and for some or all samples of $\tau^{\prime}$ and $\tau$. This will result in reduced or no resolution for those scatterers.

The dependency of the image resolution on the length of the support of the windowing function and the carrier frequency of the transmitted waveform can also be understood from the perspective of the spreading of the iso-Doppler curves. As previously described, our imaging method performs the FBP onto the iso-Doppler curves. The image resolution is accordingly closely related to the width of the spreading of the bistatic iso-Doppler curves, which, in turn, is determined by the Doppler ambiguity of the transmitted waveform, and thus,
TABLE II

Simulation Parameters Used in Five Cases

\begin{tabular}{l|cccl}
\hline Cases & $f_{0}(\mathrm{MHz})$ & $L_{\phi}(\mathrm{s})$ & $f_{\tau}(\mathrm{Hz})$ & $\tau^{\prime}(\mathrm{s})$ \\
\hline 1 & 200 & 0.1707 & 0.9667 & $\tau^{\prime}=16.5505$ \\
2 & 200 & 2.7312 & 0.9667 & $\tau^{\prime}=16.5505$ \\
3 & 20 & 0.1707 & 0.9667 & $\tau^{\prime}=16.5505$ \\
4 & 200 & 0.1707 & 1.9335 & $\tau^{\prime}=16.5505$ \\
5 & 200 & 0.1707 & 0.9667 & $\tau^{\prime}=[0,264.8086]$ \\
\hline
\end{tabular}

the duration of the signal to be processed, and the transmitter frequency.

We performed simulations to show the effects of the Doppler ambiguity and the frequency of the transmitted waveform on the width of the spreading of the iso-Doppler curves. As shown in Fig. 4, the spreading of the iso-Doppler curve becomes narrow with a longer windowing function or a higher transmitter frequency, which implies an improved image resolution.

\section{F. Reconstruction Algorithm and Its Computational Complexity}

In this section, we describe the numerical implementation of our FBP method given in (24) and (25) and analyze its computational complexity.

Let $\boldsymbol{\Xi}\left(\tau^{\prime}, \tau, \boldsymbol{z}\right)=\left(\boldsymbol{\Xi}_{1}, \boldsymbol{\Xi}_{2}\right)$ and $\partial_{\tau} \boldsymbol{\Xi}\left(\tau^{\prime}, \tau, \boldsymbol{z}\right)=\left(\dot{\boldsymbol{\Xi}}_{1}, \dot{\boldsymbol{\Xi}}_{2}\right)$. Then, by (45) and (48), we have

$$
\frac{1}{\eta\left(\boldsymbol{z}, \tau^{\prime}, \boldsymbol{\xi}\right)}=\left|\frac{\partial \boldsymbol{\xi}}{\partial(t, \tau)}\right|=|t|\left|\boldsymbol{\Xi}_{1} \dot{\boldsymbol{\Xi}}_{2}-\dot{\boldsymbol{\Xi}}_{1} \boldsymbol{\Xi}_{2}\right| .
$$

We refer to $|t|$ in (55) as the time-domain ramp filter, which has a similar effect on the reconstructed image as the wellknown ramp filter in the tomography literature [21]. The image formed without the time-domain ramp filtering has smooth edges.

We write the filter (52) as

$$
Q\left(z, t, \tau^{\prime}, \tau\right)=Q_{1}\left(z, \tau^{\prime}, \tau\right) Q_{2}\left(z, t, \tau^{\prime}, \tau\right)|t|
$$




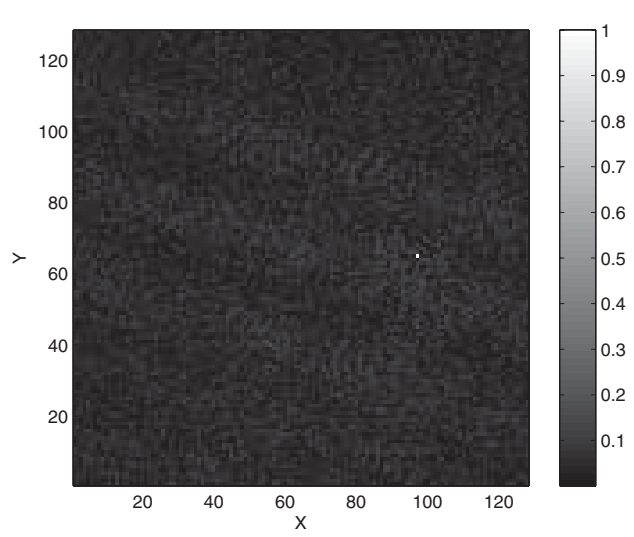

(a)

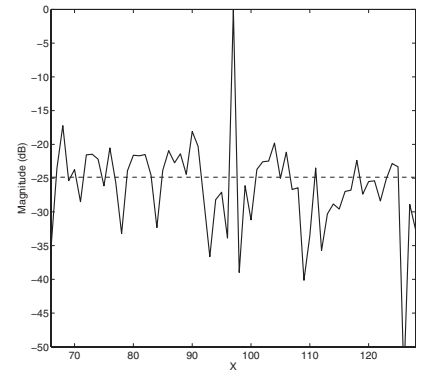

(b)

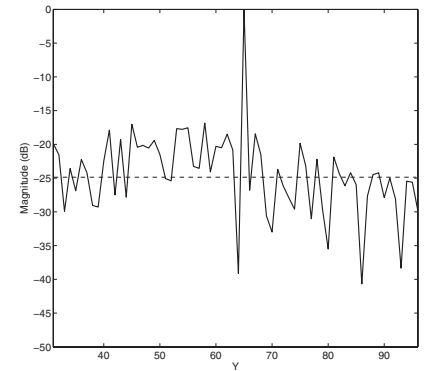

(c)
Fig. 6. (a) PSF of the DSAR imaging method at $(825,550,0) \mathrm{m}$ along with its (b) $X$ profile, and (c) $Y$ profile, for a transmitter transmitting a DAB waveform at $f_{0}=200 \mathrm{MHz}$, and the transmitter and receiver traversing circular trajectories $\gamma_{T}(s)=\gamma_{C}(s)$ and $\gamma_{R}(s)=\gamma_{C}(s-\pi / 4)$, respectively. See (65) for the explicit form of $\gamma_{C}$. The length of the support of the windowing function is $L_{\phi}=0.1707 \mathrm{~s}$. The sampling rate of $\tau$ is $f_{\tau}=$ $0.9667 \mathrm{~Hz}$.

where

$$
\begin{aligned}
Q_{1}\left(z, \tau^{\prime}, \tau\right) & =\left|\mathbf{\Xi}_{1} \dot{\boldsymbol{\Xi}}_{2}-\dot{\boldsymbol{\Xi}}_{1} \mathbf{\Xi}_{2}\right| \\
Q_{2}\left(z, t, \tau^{\prime}, \tau\right) & =\chi \Omega_{\tau^{\prime}, \boldsymbol{z}} \frac{A^{*}\left(\boldsymbol{z}, t, \tau^{\prime}, \tau\right)}{\left|A\left(\boldsymbol{z}, t, \tau^{\prime}, \tau\right)\right|^{2}} .
\end{aligned}
$$

Note that the filter $Q_{1}$ compensates the amplitude modulation due to the change of variables given in (45), while the filter $Q_{2}$ compensates the amplitude modulation terms related to the antenna beam pattern and the transmitted waveform.

Using (20), (24), (25), and (56), we obtain the following reconstruction formula:

$$
\begin{aligned}
\tilde{\rho}(\boldsymbol{z})= & \int e^{-i 2 \pi f_{0} t\left[1-\frac{f_{d}\left(\tau^{\prime}, \tau, \boldsymbol{z}\right)}{f_{0}}\right]}|t| D\left(\tau^{\prime}, \tau, t\right) \\
& \times Q_{1}\left(\boldsymbol{z}, \tau^{\prime}, \tau\right) Q_{2}\left(\boldsymbol{z}, t, \tau^{\prime}, \tau\right) d t d \tau d \tau^{\prime}
\end{aligned}
$$

where

$$
D\left(\tau^{\prime}, \tau, t\right)=\int e^{i 2 \pi f_{0} t \mu} d\left(\tau^{\prime}, \tau, \mu\right) d \mu .
$$

We assume that there are $\mathcal{O}(N)$ samples of $\mu, \tau, \tau^{\prime}, t$ variables, respectively, and that the scene is discretized into $\mathcal{O}(N \times N)$ points. The steps of the reconstruction algorithm and their corresponding computational complexity are as follows.

1) Form the Correlation Data Using (9): For each $\tau^{\prime}, \tau, \mu$ sample, the computational complexity of the correlation in

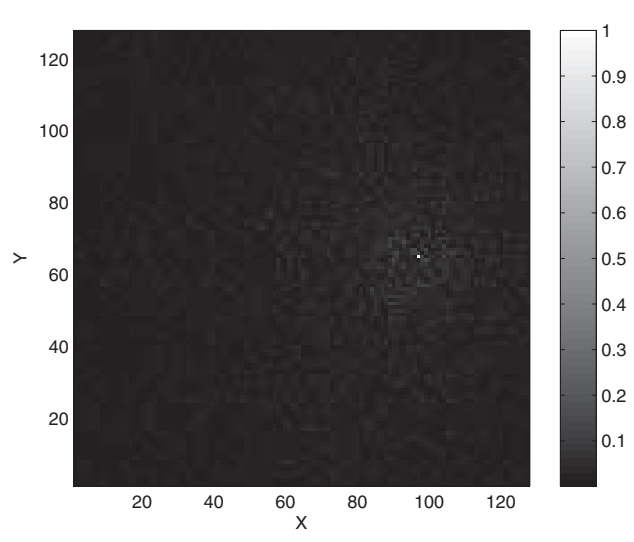

(a)

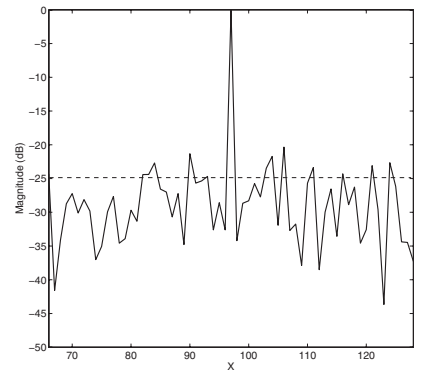

(b)

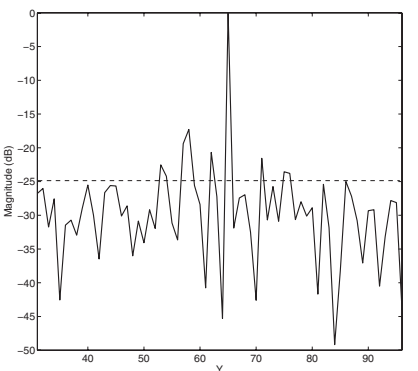

(c)
Fig. 7. (a) PSF of the DSAH imaging method at $(825,550,0) \mathrm{m}$ along with its (b) $X$ profile, and (c) $Y$ profile, for a transmitter transmitting a DAB waveform at $f_{0}=200 \mathrm{MHz}$, and the transmitter and receiver traversing circular trajectories, $\gamma_{T}(s)=\gamma_{C}(s)$ and $\gamma_{R}(s)=\gamma_{C}(s-\pi / 4)$, respectively. See (65) for the explicit form of $\gamma_{C}$. The length of the support of the windowing function is $L_{\phi}=2.7312 \mathrm{~s}$. The sampling rate of $\tau$ is $f_{\tau}=$ $0.9667 \mathrm{~Hz}$.

(9) is $\mathcal{O}(N)$. Thus, for all $\tau, \tau^{\prime}$, and $\mu$, the computational complexity of this step is $\mathcal{O}\left(N^{4}\right)$.

2) Compute the Fourier Transform of the Correlated Data With Respect to $\mu$ : For each $\tau$ and $\tau^{\prime}$, the Fourier transform of the correlated data, i.e., $d\left(\tau^{\prime}, \tau, \mu\right)$, is calculated with respect to $\mu$, as described in (60). This operation can be performed using the fast Fourier transform in $\mathcal{O}(N \log N)$ number of computations. Thus, for all $\tau$ and $\tau^{\prime}$, the computational complexity of this step is $\mathcal{O}\left(N^{3} \log N\right)$.

3) Ramp Filtering: Let

$$
\tilde{D}\left(\tau^{\prime}, \tau, t\right)=|t| D\left(\tau^{\prime}, \tau, t\right) .
$$

For each $\tau$ and $\tau^{\prime}$, (61) can be computed in $\mathcal{O}(N)$ number of computations. Thus, for all $\tau$ and $\tau^{\prime}$, the computational complexity of this step is $\mathcal{O}\left(N^{3}\right)$.

4) Filtering With $Q_{2}$ : Let

$$
\tilde{D}_{Q_{2}}\left(z, \tau^{\prime}, \tau, t\right)=\tilde{D}\left(\tau^{\prime}, \tau, t\right) Q_{2}\left(z, t, \tau^{\prime}, \tau\right)
$$

For each $\tau, \tau^{\prime}$, and $\boldsymbol{z},(62)$ can be computed in $\mathcal{O}(N)$ number of computations. Thus, for all $\tau, \tau^{\prime}$, and $z$, the computational complexity of this step is $\mathcal{O}\left(N^{5}\right)$.

5) Backprojection Step: We backproject the filtered data obtained in step (4) onto the bistatic iso-Doppler contours as 


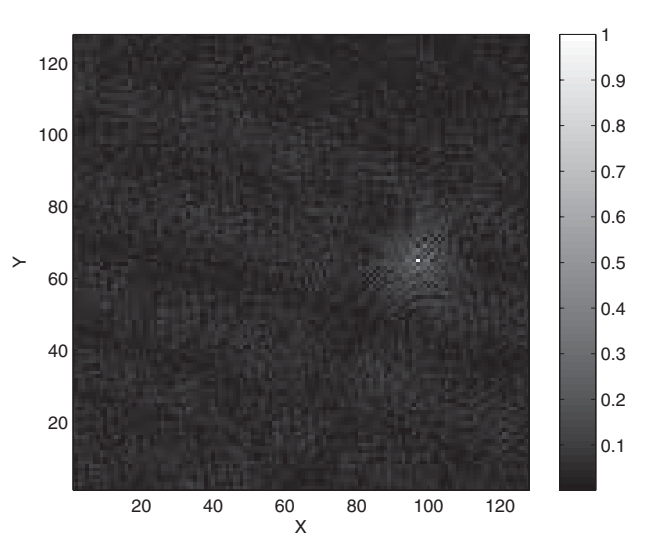

(a)

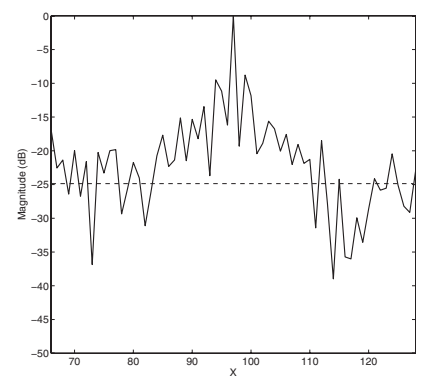

(b)

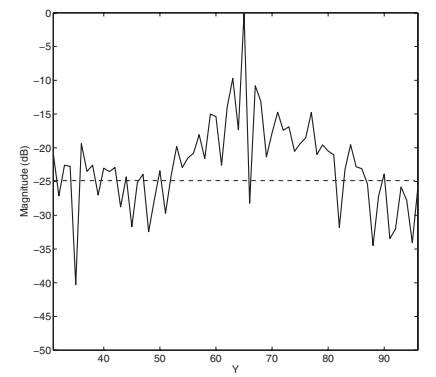

(c)
Fig. 8. (a) PSF of the DSAH imaging method at $(825,550,0) \mathrm{m}$ along with its (b) $X$ profile, and (c) $Y$ profile, for a transmitter transmitting a DAB waveform at $f_{0}=20 \mathrm{MHz}$, and the transmitter and receiver traversing circular trajectories, $\gamma_{T}(s)=\gamma_{C}(s)$ and $\gamma_{R}(s)=$ $\gamma_{C}(s-\pi / 4)$, respectively. See (65) for the explicit form of $\gamma_{C}$. The length of the support of the windowing function is $L_{\phi}=0.1707 \mathrm{~s}$. The sampling rate of $\tau$ is $f_{\tau}=0.9667 \mathrm{~Hz}$.

follows:

$$
\tilde{\rho}_{\tau^{\prime}, \tau}(z)=\int \mathrm{e}^{-\mathrm{i} 2 \pi f_{0} t\left[1-\frac{f_{d}\left(\tau^{\prime}, \tau, \boldsymbol{z}\right)}{f_{0}}\right]} \widetilde{D}_{\mathrm{Q}_{2}}\left(z, \tau^{\prime}, \tau, \mathrm{t}\right) d t .
$$

For each $\tau, \tau^{\prime}$, and $z$, (63) can be computed using direct computation in $\mathcal{O}(N)$ number of computations. Thus, for all $\tau$, $\tau^{\prime}$, and $z$, the computational complexity of this step is $\mathcal{O}\left(N^{5}\right)$.

6) Image Formation: We form the image $\tilde{\rho}(z)$ by

$$
\tilde{\rho}(\boldsymbol{z})=\int Q_{1}\left(\boldsymbol{z}, \tau^{\prime}, \tau\right) \tilde{\rho}_{\tau^{\prime}, \tau}(\boldsymbol{z}) d \tau d \tau^{\prime}
$$

The computational complexity of this step is $\mathcal{O}\left(N^{4}\right)$.

Thus, our image reconstruction algorithm has a computational complexity of $\mathcal{O}\left(N^{3} \log N\right)-\mathcal{O}\left(N^{5}\right)$ where the backprojection step determines the computational complexity of the reconstruction algorithm. Note that the computational complexity above is estimated without the use of fast backprojection algorithms [18], [31]-[33]. The efficiency of our imaging method can be further improved by utilizing fast backprojection algorithms where the computational complexity can be potentially reduced to $\mathcal{O}\left(N^{3} \log N\right)$ for omnidirectional antennas [18], [31], or by utilizing fast fourier integral operator computation methods with a computational complexity of $\mathcal{O}\left(N^{3} \log N \log (1 / \epsilon)\right)$ with $\epsilon$ being the desired pixelwise accuracy [32], [33].

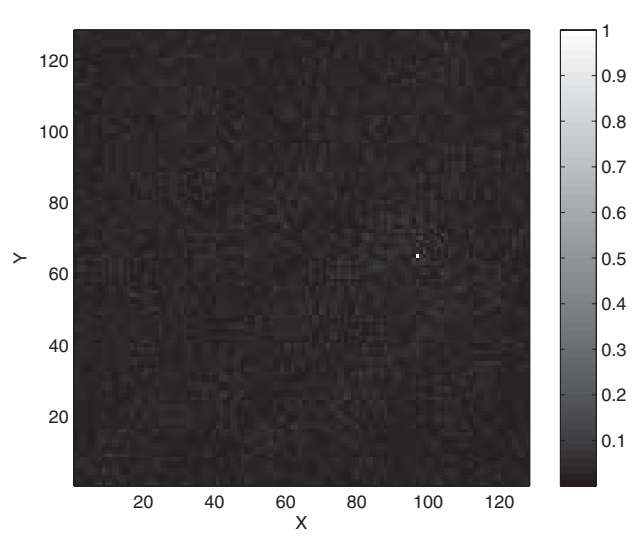

(a)

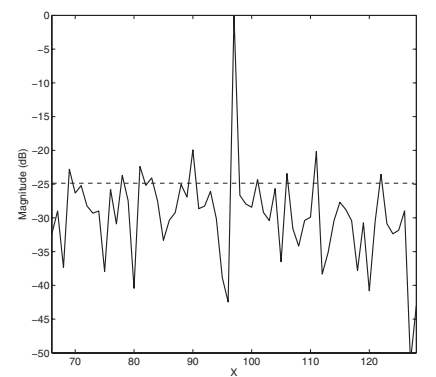

(b)

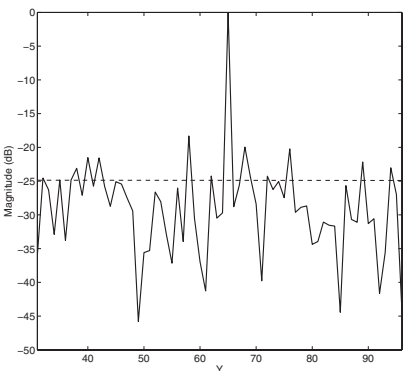

(c)
Fig. 9. (a) PSF of the DSAH imaging method at $(825,550,0) \mathrm{m}$ along with its (b) $X$ profile, and (c) $Y$ profile, for a transmitter transmitting a DAB waveform at $f_{0}=200 \mathrm{MHz}$, and the transmitter and receiver traversing circular trajectories, $\gamma_{T}(s)=\gamma_{C}(s)$ and $\gamma_{R}(s)=\gamma_{C}(s-\pi / 4)$, respectively. See (65) for the explicit form of $\gamma_{C}$. The length of the support of the windowing function is $L_{\phi}=0.1707 \mathrm{~s}$. The sampling rate of $\tau$ is $f_{\tau}=1.9335 \mathrm{~Hz}$.

\section{Numerical Simulations}

We conducted two sets of numerical simulations: 1) we numerically studied the PSF of the imaging operator under different scenarios and demonstrated the theoretical results described in Section III-E and 2) we demonstrated the performance of our imaging method for extended targets. Note that noise is not considered in our simulation. However, our imaging method is also applicable to the case where the raw data is corrupted by additive noise [25], [34].

We used the digital audio broadcasting (DAB) signals used by radio stations as the transmitted waveform in the numerical simulations. We simulated the DAB signal based on the European standard, which uses the coded orthogonal frequency division multiplexing modulation. The main characteristics of the DAB signals are as follows: 1) the symbol has $1 \mathrm{~ms}$ useful duration with a guard interval of $0.246 \mathrm{~ms}$; 2) per symbol, 1536 subcarriers are transmitted simultaneously; and 3) quadrature phase shift keying coding is used for each subcarrier. The bandwidth of the DAB signal is $1.5 \mathrm{MHz}$. In the simulations, the transmitter was assumed to be operating in the very high frequency band at a transmission frequency of $200 \mathrm{MHz}$.

We considered a scene of size $[0,1100] \times[0,1100] \mathrm{m}^{2}$ with flat topography. The scene was discretized into $128 \times 128$ 
TABLE III

3-dB Main Lobe Width And PSLR of the Reconstructed PSFs For CASES 1-4

\begin{tabular}{l|cc|cc}
\hline & \multicolumn{2}{|c}{$X$ direction } & \multicolumn{2}{c}{$Y$ direction } \\
Cases $\left(f_{0}, L_{\phi}, f_{\tau}\right)$ & 3-dB main lobe width $(\mathrm{m})$ & PSLR $(\mathrm{dB})$ & 3-dB main lobe width $(\mathrm{m})$ & PSLR $(\mathrm{dB})$ \\
\hline$(1)(200 \mathrm{MHz}, 0.1707 \mathrm{~s}, 0.9667 \mathrm{~Hz})$ & 1.7253 & -14.1168 & 1.9221 & -16.8394 \\
$(2)(200 \mathrm{MHz}, 2.7312 \mathrm{~s}, 0.9667 \mathrm{~Hz})$ & 1.5434 & -20.3416 & 1.3767 & -17.2813 \\
$(3)(20 \mathrm{MHz}, 0.1707 \mathrm{~s}, 0.9667 \mathrm{~Hz})$ & 2.9305 & -8.7848 & 2.4045 & -9.7147 \\
$(4)(200 \mathrm{MHz}, 0.1707 \mathrm{~s}, 1.9335 \mathrm{~Hz})$ & 1.6744 & -19.9312 & 1.8531 & -18.0371 \\
\hline
\end{tabular}

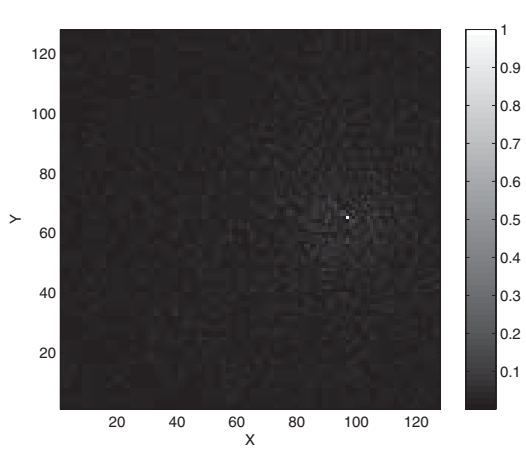

(a)

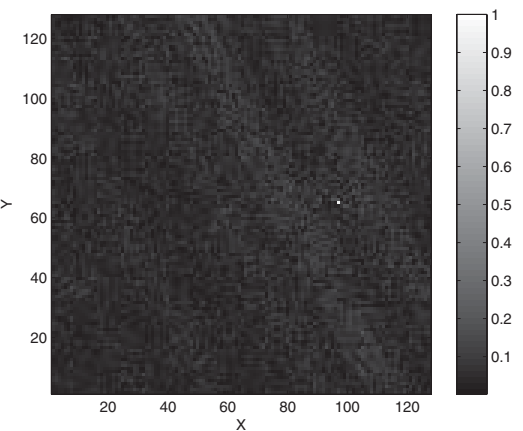

(b)

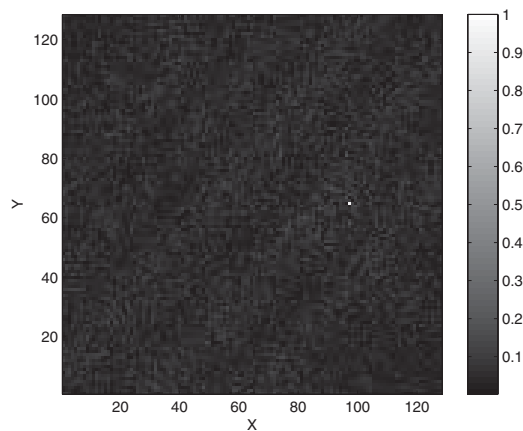

(c)

Fig. 10. (a) PSF at $[825,550,0] \mathrm{m}$ with $16 \tau^{\prime}$ values uniformly spaced in $[0,264.8086] \mathrm{s}$ and the PSFs reconstructed using the vectors in the partial data collection manifolds. (b) $\Omega_{\tau^{\prime}=115.8538 \mathrm{~s}}$. (c) $\Omega_{\tau^{\prime}=198.6064 \mathrm{~s}}$, with a transmitter transmitting a DAB waveform at $f_{0}=200 \mathrm{MHz}$. The transmitter and receiver were traversing circular flight trajectories $\gamma_{T}(s)$ and $\gamma_{R}(s)$, respectively, as shown in Fig. 5. The length of the support of the windowing function is $L_{\phi}=0.1707 \mathrm{~s}$. The sampling rate of $\tau$ is $f_{\tau}=0.9667 \mathrm{~Hz}$.

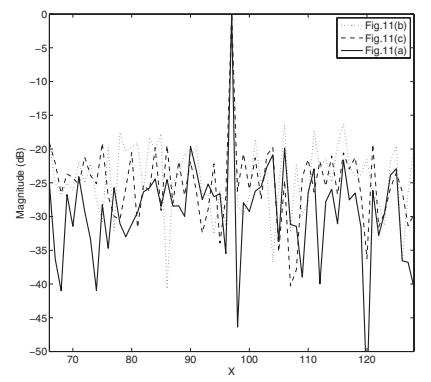

(a)

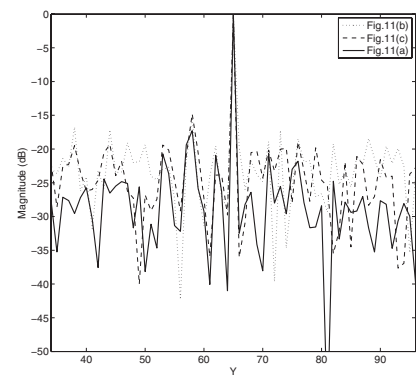

(b)
Fig. 11. (a) $X$ and (b) $Y$ profiles of the reconstructed PSFs shown in Fig. 10.

pixels, where $[0,0,0] \mathrm{m}$ and $[1100,1100,0] \mathrm{m}$ correspond to the pixels $(1,1)$ and $(128,128)$, respectively.

In all the numerical simulations, we assumed that both the receiver and transmitter antennas were isotropic. We assumed that the transmitter and the receiver were traversing the circular trajectory given by

$$
\gamma_{C}(s)=(11+11 \cos (s), 11+11 \sin (s), 6.5) \mathrm{km} .
$$

Let $\boldsymbol{\gamma}_{T}(s)$ and $\boldsymbol{\gamma}_{R}(s)$ denote the trajectories of the transmitter and receiver, respectively. We set $\boldsymbol{\gamma}_{T}(s)=\boldsymbol{\gamma}_{C}(s)$ and $\boldsymbol{\gamma}_{R}(s)=$ $\gamma_{C}(s-(\pi / 4))$. Note that the variable $s$ in $\gamma_{C}$ is equal to $(v / R) t$, where $v$ is the speed of the receiver, and $R$ is the radius of the circular trajectory. We set the speed of the transmitter and receiver to $261 \mathrm{~m} / \mathrm{s}$.

For all the numerical experiments, we used (7) to generate the received signal and (9) to generate the data used for imaging and chose the windowing function $\phi$ in (9) to be a Hanning function.

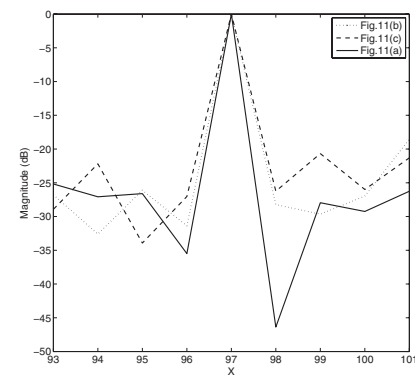

(a)

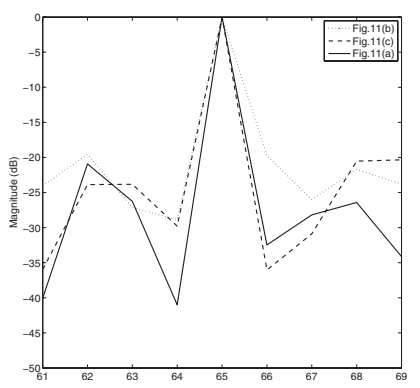

(b)
Fig. 12. Enlarged views of the main lobe region of (a) $X$ profiles and (b) $Y$ profiles of the reconstructed PSFs shown in Fig. 10.

We performed image reconstruction for each $\tau^{\prime}$ and coherently superimposed the reconstructed images obtained over a range of $\tau^{\prime}$ values.

\section{A. Numerical Analysis of the PSF}

We placed a point target with unit reflectivity at $[825,550,0] \mathrm{m}$ in the scene considered. Note that this position corresponds to the $(97,65)$ th pixel in the reconstructed scene. Fig. 5 shows the 3-D view of the scene with the target, transmitter, and receiver trajectories.

We performed numerical simulations to demonstrate the effect of $L_{\phi}$, the length of the support of $\phi(t), f_{0}$, the carrier frequency of the transmitted waveform, $f_{\tau}$, the sampling rate of $\tau$, i.e., the sampling rate of the aperture, and the range of $\tau^{\prime}$ on the PSF of the imaging operator. We reconstructed the PSF for five cases, as listed in Table II. For the fifth case, we reconstructed the PSF using $16 \tau^{\prime}$ values uniformly 
TABLE IV

3-dB Main Lobe Width ANd PSLR of the Reconstructed PSFs For DEMONSTRATING THe Superposition EFFECT

\begin{tabular}{c|cc|cc}
\hline & \multicolumn{2}{|c|}{ X direction } & \multicolumn{2}{c}{$Y$ direction } \\
Cases & 3-dB main lobe width $(\mathrm{m})$ & PSLR $(\mathrm{dB})$ & 3-dB main lobe width $(\mathrm{m})$ & PSLR $(\mathrm{dB})$ \\
\hline Superposition over $16 \tau^{\prime}$ & 1.3142 & -19.6241 & 1.4223 & -17.2785 \\
A fixed $\tau^{\prime}=115.8538 \mathrm{~s}$ & 1.7372 & -16.2563 & 2.2052 & -15.3491 \\
A fixed $\tau^{\prime}=198.6064 \mathrm{~s}$ & 1.9396 & -17.581 & 1.6847 & -14.7989 \\
\hline
\end{tabular}

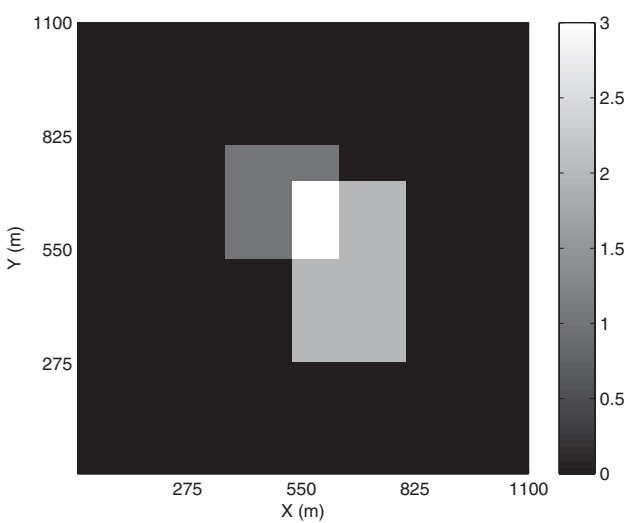

(a)

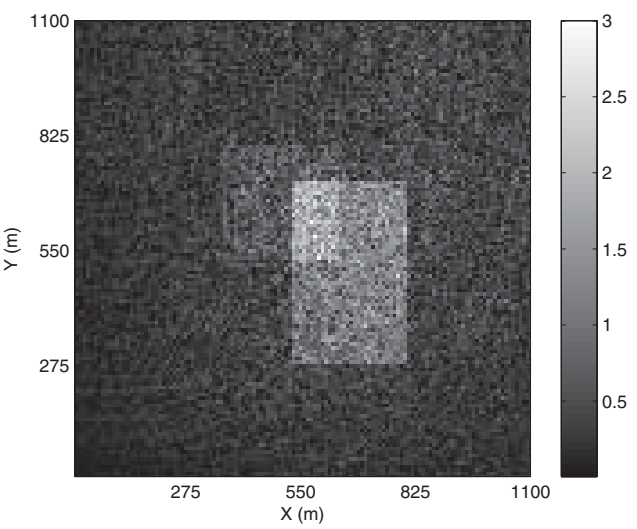

(c)

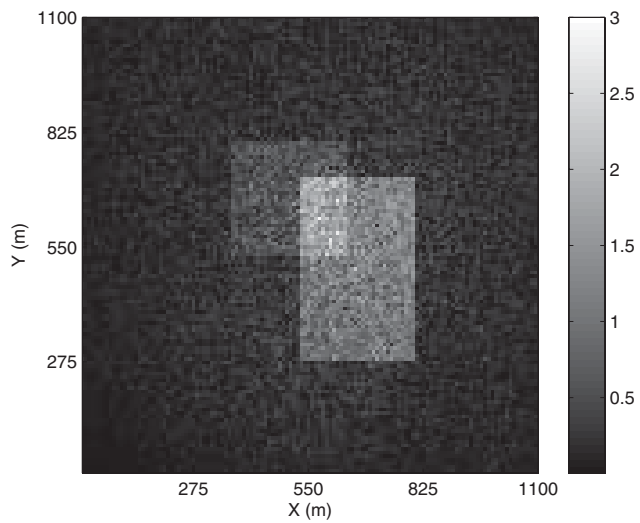

(b)

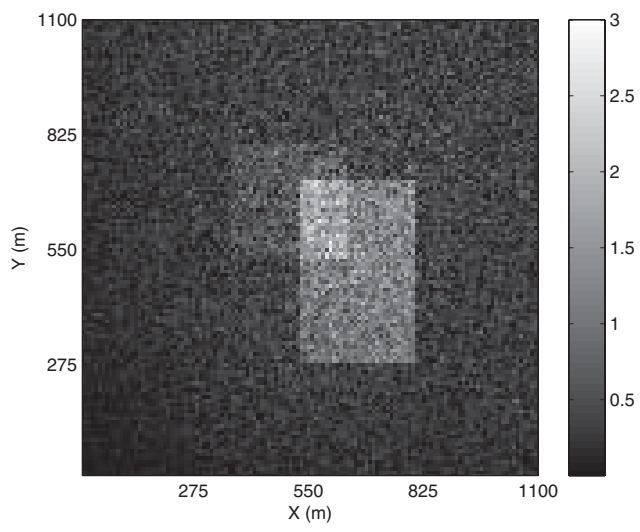

(d)

Fig. 13. (a) Scene with extended targets used in the numerical simulations. The reflectivity of the square and rectangular targets is 1 and 2 , respectively in the nonoverlapping region and 3 in the overlapping region. (b) Image reconstructed with $16 \tau^{\prime}$ values uniformly spaced in [0, 264.8086] s. The images reconstructed using the vectors in the partial data collection manifolds (c) $\Omega_{\tau^{\prime}=121.3706 \mathrm{~s}}$ and (d) $\Omega_{\tau^{\prime}=237.2243 \mathrm{~s}}$. The transmitter was assumed to be transmitting a DAB waveform at $f_{0}=200 \mathrm{MHz}$. The transmitter and receiver were traversing circular flight trajectories $\boldsymbol{\gamma}_{T}(s)$ and $\boldsymbol{\gamma}_{R}(s)$, respectively, as shown in Fig. 5 . The length of the support of the windowing function is $L_{\phi}=2.7312 \mathrm{~s}$. The sampling rate of $\tau$ is $f_{\tau}=3.8668 \mathrm{~Hz}$.

spaced in $[0,264.8086] \mathrm{s}$. Note that $f_{\tau}=0.9667 \mathrm{~Hz}$ and $f_{\tau}=1.9335 \mathrm{~Hz}$ correspond to the uniform sampling of the circular trajectory with 256 and 512 points, respectively.

Figs. 6-9 show the reconstructed PSFs along with their profiles in the $X$ and $Y$ directions for the first four cases. The axes of the figures are labeled according to the pixel number. Note that this convention is used for displaying all the reconstructed images in this subsection. We also tabulated the 3-dB main lobe width and the peak-to-side lobe ratio (PSLR) of the $X$ and $Y$ profiles in Table III, where the 3-dB main lobe width is used as a measure of resolution and PSLR is used as a measure of the level of the side lobes in the reconstructed PSFs.
As can be seen in Figs. 6 and 7, the quality of the PSF improves as the length of the support of the windowing function increases from 0.1707 to $2.7312 \mathrm{~s}$ due to a larger data collection manifold. Comparing the profiles of the PSFs in both cases, we see that the level of the side lobes of the PSF decreases as the support of windowing function gets longer, which is also indicated by the PSLR values in the two cases listed in Table III. This results in an increase of the image contrast, as shown in Fig. 7.

Note that the change in the resolution is not evident visually when the images in Figs. 6 and 7 are compared. This may be due to the relatively large pixel size of the reconstructed image. 
We can clearly see in Table IV that the resolution in both the $X$ direction and $Y$ direction improves.

Comparing Fig. 8 with Fig. 6, we see that the quality of the reconstructed PSF degrades because of the decrease in the frequency of the transmitted waveform from 200 to $20 \mathrm{MHz}$ as indicated by the theory. We observe a spreading of the main lobes and an obvious increase in the level of the side lobes in Fig. 8(b) and (c). This is also indicated by the increase in the 3-dB main lobe width and the PSLR as shown in Table III.

Comparing Fig. 9 with Fig. 6, we see that the increase in the sampling rate of $\tau$, which implies larger number of $\tau$ samples, results in an improvement in the reconstructed PSF. Specifically, we observe that there is a significant improvement in the suppression of the side lobes of the PSF as shown in Fig. 9(a) and (b), which is also indicated by the decrease of the PSLR value from cases 1-4 in Table III. Furthermore, we see from Table III that the resolutions in the $X$ and $Y$ directions improve moderately, although the change of the resolution is not visually noticeable when the images in Figs. 6(a) and 9(a) are compared.

Fig. 10 shows the reconstructed PSF for the fifth case as listed in Table III, which is the superposition of the images obtained for 16 different $\tau^{\prime}$ values uniformly spaced in $[0,264.8086] \mathrm{s}$. We use this simulation to demonstrate the effect of the superposition of images reconstructed for each $\tau^{\prime}$. To compare the effect of the superposition of the reconstructed images and the value of $\tau^{\prime}$, we present the reconstructed images corresponding to two different values of $\tau^{\prime}$ in Fig. 10(b) and (c) and the profiles of the reconstructed PSFs in $X$ and $Y$ directions together in Fig. 11. Fig. 12 presents the enlarged views of the mainlobe region. Table IV includes the 3-dB main lobe width and PSLR of the $X$ and $Y$ profiles.

As expected, since the partial data collection manifold $\Omega_{\tau^{\prime}, \boldsymbol{z}}$ for a fixed $\tau^{\prime}$ is significantly smaller than $\cup_{\tau^{\prime}} \boldsymbol{\Omega}_{\tau^{\prime}, \boldsymbol{z}}$, the quality of the images in Fig. 10(b) and (c) is poorer than that of the superimposed image in Fig. 10(a). As shown in Fig. 11 and indicated by the PSLR values in Table IV, the superposition suppresses the side lobes significantly. This can be also observed visually in Fig. 10. Furthermore, as indicated by Fig. 12 and the 3-dB main lobe width tabulated in Table III, the resolution is improved by the superposition of the images as well.

\section{B. Numerical Simulations for Extended Targets}

We considered a square target of size $275 \mathrm{~m}$ and a rectangular target of size $275 \mathrm{~m} \times 438 \mathrm{~m}$ with their centers at $(498.4,661.7) \mathrm{m}$ and $(661.7,498.4) \mathrm{m}$, which correspond to the pixels $(58,77)$ and $(77,58)$, respectively. The reflectivity of the two targets are set to be 1 and 2, respectively. Fig. 13 shows the scene with the two partially overlapped extended targets. Note that the reflectivity of the scene in the overlapping region is 3 .

We reconstructed the targets using the DAB waveform with $L_{\phi}=2.7312 \mathrm{~s}$ and $f_{\tau}=3.8668 \mathrm{~Hz}$. The reconstructed image is shown in Fig. 13, which is the superposition of the images obtained for 16 different $\tau^{\prime}$ values uniformly spaced in $[0,264.8086] \mathrm{s}$. The two images corresponding to the fixed $\tau^{\prime}$ values are shown in Fig. 13(c) and (d).

As expected, due to a smaller data collection manifold, the quality of the images in Fig. 13(c) and (d) is poorer than that of the image obtained by the superposition of all the images reconstructed for a range of $\tau^{\prime}$ values, as shown in Fig. 13(b). We observe that the images are more noisy in Fig. 13(c) and (d) and the targets, particularly the square target with relatively weak reflectivity, do not appear as sharp as the ones in Fig. 13(b).

\section{CONCLUSION}

We presented a novel imaging method for bistatic SAR using ultranarrowband waveforms. Our imaging method consists of a novel forward model and a corresponding FBP-type novel image formation method. The model was formed by the windowed, scaled (or frequency shifted), and translated correlations of the received signal with the transmitted signal. The image formation was developed using microlocal techniques which resulted in an FBP-type inversion of the forward model.

High-frequency analysis of the forward model shows that the correlated data is the projection of the ground reflectivity onto bistatic iso-Doppler curves. We reconstructed the ground reflectivity by the FBP of the correlated signals onto bistatic iso-Doppler curves.

The analysis of the PSF of the imaging operator shows that the visible singularities of the scene are those that are at the intersection of bistatic iso-Doppler curves and bistatic iso-Doppler rate curves. Furthermore, the FBP-type inversion puts the visible edges of the scene at the correct location and orientation with the correct strength.

Our analysis showed that the resolution of the reconstructed image is primarily determined by the temporal duration of the signal used for imaging and the carrier frequency of the transmitted waveform, which is consistent with the ambiguity theory of the CW or ultranarrowband waveforms. Additionally, our resolution analysis identified various other factors, such as the velocity of the antennas and the range of the antennas to the scene that affect the resolution of reconstructed images.

We described the algorithmic implementation and computational complexity of our reconstruction method and presented extensive numerical experiments using DAB signals to validate the theoretical analysis and to demonstrate the performance of our imaging method.

Although our imaging scheme was developed in a deterministic setting, it is also applicable when the measurements are corrupted by additive white Gaussian noise [34]. When $a$ priori information for the scene to be reconstructed is available and additive noise is colored, FBP-type inversion method presented in this paper can be extended as described in [25].

Our imaging technique is particularly suitable for passive synthetic aperture imaging using cooperative sources of opportunity-transmitting ultranarrowband waveforms.

The presented method is not restricted to a particular geometry. It can be used for arbitrary trajectories, non-flat topography, and stationary or mobile transmitters. Furthermore, our image reconstruction is analytic. Therefore, it can be implemented fast [18], [31]-[33]. 


\section{REFERENCES}

[1] D. Mensa, S. Halevy, and G. Wade, "Coherent doppler tomography for microwave imaging," Proc. IEEE, vol. 71, no. 2, pp. 254-261, Feb. 1983.

[2] D. Mensa and G. Heidbreder, "Bistatic synthetic-aperture radar imaging of rotating objects," IEEE Trans. Aerosp. Electron. Syst., vol. 18, no. 4, pp. 423-431, Jul. 1982.

[3] H. Sun, H. Feng, and Y. Lu, "High resolution radar tomographic imaging using single-tone CW signals," in Proc. IEEE Radar Conf., May 2010, pp. 975-980.

[4] M. C. Wicks, B. Himed, J. L. E. Bracken, H. Bascom, and J. Clancy, "Ultranarrow band adaptive tomographic radar," in Proc. IEEE Int. Workshop Comput. Adv. Multi-Sensor Adapt. Process., Dec. 2005, pp. 36-39.

[5] S. L. Coetzee, C. J. Baker, and H. D. Griffiths, "Narrow band high resolution radar imaging," in Proc. IEEE Radar Conf., Apr. 2006, pp. $622-625$.

[6] J. H. Thomson and J. E. B. Ponsonby, "2-D aperture synthesis in lunar radar astronomy," Proc. Royal Soc. London Ser. A, vol. 303, no. 1475, pp. 477-491, 1968.

[7] M. S. Roulston and D. O. Muhleman, "Synthesizing radar maps of polar regions with a doppler-only method," Appl. Opt., vol. 36, pp. 3912-3919, Jun. 1997.

[8] B. Borden and M. Cheney, "Synthetic-aperture imaging from highdoppler-resolution measurements," Inverse Problems, vol. 21, pp. 1-11, Nov. 2005.

[9] C. E. Yarman, L. Wang, and B. Yazıcı, "Doppler synthetic aperture hitchhiker imaging," Inverse Problems, vol. 26, no. 6, pp. 065006-1065006-26, 2010.

[10] H. D. Griffiths and C. J. Baker, "Passive coherent location radar systems. Part 1: Performance prediction," IEE Proc.-Radar Sonar Nav., vol. 152 , no. 3, pp. 153-159, Jun. 2005.

[11] P. E. Howland, D. Maksimiuk, and G. Reitsma, "FM radio based bistatic radar," IEE Proc.-Radar, Sonar Nav., vol. 152, no. 3, pp. 107-115, Jun. 2005.

[12] D. Tan, H. Sun, Y. Lu, M. Lesturgie, and H. L. Chan, "Passive radar using global system for mobile communication signal: Theory, implementation and measurements," IEE Proc.-Radar Sonar Nav., vol. 152, no. 3, pp. 116-123, Jun. 2005.

[13] D. Poullin, "Passive detection using digital broadcasters (DAB, DVB) with COFDM modulation," IEE Proc.-Radar Sonar Nav., vol. 152, no. 3 , pp. 143-152, Jun. 2005.

[14] D. W. O'Hagan and C. J. Baker, "Passive bistatic radar (PBR) using FM radio illuminators of opportunity," in Proc. IEEE Radar Conf., Rome, Italy, May 2008, pp. 1-6.

[15] C. Coleman and H. Yardley, "Passive bistatic radar based on target illuminations by digital audio broadcasting," IET Radar Sonar Nav., vol. 2, no. 5, pp. 366-375, Oct. 2008.

[16] K. Chetty, K. Woodbridge, H. Guo, and G. E. Smith, "Passive bistatic WiMAX radar for marine surveillance," in Proc. IEEE Radar Conf., Washington, DC, May 2010, pp. 188-193.

[17] P. Falcone, F. Colone, C. Bongioanni, and P. Lombardo, "Experimental results for OFDM WiFi-based passive bistatic radar," in Proc. IEEE Radar Conf., Washington, DC, May 2010, pp. 516-521.

[18] S. Nilsson, "Application of fast backprojection techniques for some inverse problems of integral geometry," Ph.D. dissertation, Linköping Studies Science Technology, Linköping University, Linköping, Sweden, 1997.

[19] C. J. Nolan and M. Cheney, "Synthetic aperture inversion for arbitrary flight paths and nonflat topography," IEEE Trans. Image Process., vol. 12, no. 9, pp. 1035-1043, Sep. 2003.

[20] J. J. Duistermaat, Fourier Integral Operators. Boston, MA: Birkhauser, 1996.

[21] F. Natterer and F. Wübbeling, Mathematical Methods in Image Reconstruction. Philadelphia, PA: SIAM, 2001.

[22] N. Bleistein and R. Handelsman, Asymptotic Expansions of Integrals. New York: Dover, 1986.

[23] A. Grigis and J. Sjöstrand, Microlocal Analysis for Differential Operators: An Introduction (London Mathematical Society Lecture Note), vol. 196. Cambridge, U.K.: Cambridge Univ. Press, 1994.

[24] F. Treves, Introduction to Pseudodifferential and Fourier Integral Operators, vols. 1-2. New York: Plenum Press, 1980.

[25] B. Yazici, M. Cheney, and C. E. Yarman, "Synthetic-aperture inversion in the presence of noise and clutter," Inverse Problems, vol. 22, no. 5, pp. 1705-1729, 2006.
[26] C. J. Nolan and M. Cheney, "Synthetic aperture inversion," Inverse Problems, vol. 18, no. 1, pp. 221-236, 2002.

[27] E. T. Quinto, "Singularities of the X-ray transform and limited data tomography in $r^{2}$ and $r^{3}$," SIAM J. Math. Anal., vol. 24, no. 5, pp. $1215-1225,1993$.

[28] R. Courant and F. John, Introduction to Calculus and Analysis, vols. 1-2. New York: Springer-Verlag, 2000.

[29] C. J. Nolan and M. Cheney, "Microlocal analysis of synthetic aperture radar imaging," J. Fourier Anal. Appl., vol. 10, no. 2, pp. 133-148, 2004.

[30] C. E. Yarman, B. Yazıcı, and M. Cheney, "Bistatic synthetic aperture radar imaging for arbitrary flight trajectories," IEEE Trans. Image Process., vol. 17, no. 1, pp. 84-93, Jan. 2008.

[31] L. M. H. Ulander, H. Hellsten, and G. Stenström, "Syntheticaperture radar processing using fast factorized back-projection," IEEE Trans. Aerosp. Electron. Syst., vol. 39, no. 3, pp. 760-776, Jul. 2003.

[32] E. Candès, L. Demanet, and L. Ying, "Fast computation of Fourier integral operators," SIAM J. Sci. Comput., vol. 29, no. 6, pp. 2463-2493, 2007.

[33] E. Candès, L. Demanet, and L. Ying, "A fast butterfly algorithm for the computation of Fourier integral operators," SIAM Multiscale Model. Simul., vol. 7, no. 4, pp. 1727-1750, 2009.

[34] K. Voccola, B. Yazici, M. Cheney, and M. Ferrara, "On the equivalence of the generalized likelihood ratio test and backprojection method in synthetic aperture imaging," in Proc. Defense Secur. Conf., vol. 7335. Orlando, FL, Apr. 2009, pp. 0I-1-0I-10.

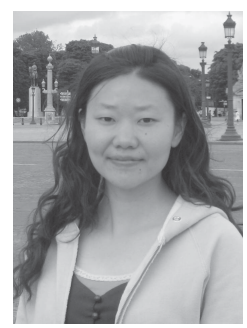

Ling Wang (M'07) received the B.S. degree in electrical engineering and the M.S. and Ph.D. degrees in information acquirement and processing from the Nanjing University of Aeronautics and Astronautics, Nanjing, China, in 2000, 2003, and 2006, respectively.

She has been with the Nanjing University of Aeronautics and Astronautics since 2003, where she is currently an Associate Professor with the Department of Information and Communication Engineering. From February 2008 to May 2009, she was a Post-Doctoral Research Associate with the Department of Mathematical Sciences and the Department of Electrical, Computer, and Systems Engineering, Rensselaer Polytechnic Institute, Troy, NY. Her current research interests include inverse scattering, wave-based imaging, radar imaging, and passive imaging.

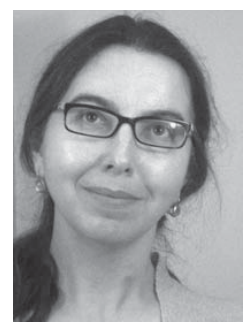

Birsen Yazici (SM'05) received the B.S. degree in electrical engineering and mathematics from Bogazici University, Istanbul, Turkey, in 1988, and the M.S. and Ph.D. degrees in mathematics and electrical engineering from Purdue University, West Lafayette, IN, in 1990 and 1994, respectively.

She was a Research Engineer with the General Electric Company Global Research Center, Schenectady, NY, from September 1994 to 2000. During her tenure in industry, she worked on radar, transportation, and industrial and medical imaging systems. From 2001 to 2003, she was an Assistant Professor with the Department of Electrical and Computer Engineering, Drexel University, Philadelphia, PA. Since 2003, she has been with Rensselaer Polytechnic Institute, Troy, NY, where she is currently a Professor with the Departments of Electrical, Computer, and Systems Engineering and Biomedical Engineering. She holds 11 U.S. patents. Her current research interests include statistical signal processing, inverse problems in imaging, image reconstruction, biomedical optics, radar, and X-ray imaging.

Dr. Yazici currently serves as an Associate Editor for the IEEE TRANSACTIONS ON IMAGE PROCESSING and the SIAM Journal on Imaging Science. Her work on industrial systems received the second Best Paper Award from the IEEE TRANSACTIONS ON INDUSTRIAL APPLICATIONS in 1997. She was the recipient of the Rensselaer Polytechnic Institute School of Engineering Research Excellence Award in 2007. 